\title{
CONVERGENCE OF A FINITE ELEMENT DISCRETIZATION OF THE NAVIER-STOKES EQUATIONS IN VORTICITY AND STREAM FUNCTION FORMULATION
}

\author{
Mohamed Amara $^{1}$ And Christine Bernardi ${ }^{2}$
}

\begin{abstract}
The standard discretization of the Stokes and Navier-Stokes equations in vorticity and stream function formulation by affine finite elements is known for its bad convergence. We present here a modified discretization, we prove that the convergence is improved and we establish a priori error estimates.

Résumé. Il est bien connu que la discrétisation usuelle des équations de Stokes et de Navier-Stokes en formulation tourbillon et fonction courant par éléments finis affines converge mal. Nous présentons ici une discrétisation modifiée dont nous prouvons la meilleure convergence et nous établissons des majorations d'erreur a priori.
\end{abstract}

AMS Subject Classification. 65N30.

Received: May 1998.

\section{INTRODUCTION}

When set in a two-dimensional bounded domain, the Stokes and Navier-Stokes equations admit an equivalent variational formulation where the unknowns are the stream function and vorticity. The main interest of this formulation is that only two scalar unknowns are involved instead of three, so that it could lead to very cheap discretizations. However, even in the simplest case of the linear Stokes problem, the less expensive finite element method, which relies on this formulation and the approximation by piecewise affine functions, presents poor convergence properties. From a theoretical point of view, non optimal convergence results are proven with some rather restrictive assumptions on the geometry and the mesh: they only hold in the case of a convex domain provided with a uniformly regular family of triangulations. Moreover these properties are too weak to treat the nonlinear Navier-Stokes equations. Numerical experiments confirm this lack of accuracy. A first improvement of this technique is proposed by Amara and El Dabaghi [2], however it seems unsufficient for the extension to the Navier-Stokes equations.

The aim of this paper is to present and analyze a modified version of the usual discretization by affine finite elements, which improves its convergence properties. The key idea relies on the decomposition of the vorticity as the sum of a harmonic part and another term which satisfies homogeneous Dirichlet boundary conditions. Such a decomposition is involved in the well-known Glowinski and Pironneau algorithm [13] for solving the linear

Keywords and phrases. Navier-Stokes equations, finite elements.

1 IPRA - LMA (UPRESA 5033 du CNRS), Université de Pau, avenue de l'Université, 64000 Pau, France.

2 Analyse Numérique, CNRS and Université Pierre et Marie Curie, B.C. 187, 4 place Jussieu, 75252 Paris Cedex 05, France.

(C) EDP Sciences, SMAI 1999 
system which results from the discretization. And the modification consists in adding a further stabilization term in the discrete problem, which, in contrast to [2], only concerns the harmonic part of the vorticity.

Working with this improvement, we are in a position to prove, for the Stokes problem, some quasi-optimal error estimates, with much weaker restrictions than for the standard technique. The resulting convergence property turns out to be sufficient to handle the convection term in the Navier-Stokes equations thanks to the theorem of Brezzi, Rappaz and Raviart [8]: we prove the same estimates for these equations without any further limitation on the geometry. Finally, we extend the new discretization to the case of the mortar element technique, with similar results, and we present analogous modifications of higher order discretizations that also improve the error estimates.

An outline of the paper is as follows:

- In Section 2, we recall the stream function and vorticity formulation of the Stokes problem, we describe the corresponding discrete problem and we check its well-posedness.

- In Section 3, we prove the convergence of the solution of the discrete problem towards the exact one, together with a priori error estimates.

- In Section 4, we describe the analogous discrete problem for the nonlinear Navier-Stokes equations and we prove that all the previous results still hold in this case.

- Section 5 is devoted to some further extensions: mortar element discretization, higher order discretizations.

\section{The Stokes Continuous And Discrete PROBlems}

Let $\Omega$ be a bounded two-dimensional domain with a Lipschitz-continuous boundary. For simplicity, we assume that $\Omega$ is simply-connected. In this section, we consider the Stokes problem:

$$
\begin{cases}-\Delta \boldsymbol{u}+\operatorname{grad} p=\boldsymbol{f} & \text { in } \Omega, \\ \operatorname{div} \boldsymbol{u}=0 & \text { in } \Omega, \\ \boldsymbol{u}=\mathbf{0} & \text { on } \partial \Omega,\end{cases}
$$

where the unknowns are the velocity $\boldsymbol{u}$ and the pressure $p$. The data are a density of body forces $\boldsymbol{f}$ and, only for simplicity, we take homogeneous boundary conditions on the velocity.

To write the vorticity and stream function formulation of this problem, we firstly observe that the divergencefree constraint in (2.1) is equivalent to the existence of a (scalar) stream function $\psi$ such that $\boldsymbol{u}=\operatorname{curl} \psi$. Also the nullity conditions on $\boldsymbol{u}$ on the boundary can be translated as nullity conditions on $\psi$ and its normal derivative $\partial_{n} \psi$. Finally we introduce the vorticity $\omega$ such that $\omega=$ curl $\boldsymbol{u}$. This leads to the system:

$$
\begin{cases}-\Delta \omega=\operatorname{curl} f & \text { in } \Omega, \\ -\Delta \psi=\omega & \text { in } \Omega, \\ \psi=\partial_{n} \psi=0 & \text { on } \partial \Omega .\end{cases}
$$

\subsection{The continuous problem}

Throughout this paper, we use the standard Sobolev spaces $H^{s}(\mathcal{O}), s \in \mathbb{R}$, on any two-dimensional domain $\mathcal{O}$ with a Lipschitz boundary, and the analogous spaces on any part of its boundary $\partial \mathcal{O}$. We also use the non-Hilbertian Sobolev spaces $W^{m, p}(\mathcal{O})$ of integral order $m$, provided with their usual norms and seminorms. We introduce the space that is needed for problem (2.2) (see [5]):

$$
X=\left\{\theta \in L^{2}(\Omega) ; \Delta \theta \in H^{-1}(\Omega)\right\},
$$

it is provided with the natural norm

$$
\|\theta\|_{X}=\left(\|\theta\|_{L^{2}(\Omega)}^{2}+\|\Delta \theta\|_{H^{-1}(\Omega)}^{2}\right)^{\frac{1}{2}},
$$


and we set: $M=H_{0}^{1}(\Omega)$.

Now it is readily checked that, for any $\boldsymbol{f}$ in $L^{2}(\Omega)^{2}$, problem (2.2) admits the following equivalent variational formulation: find a pair $(\omega, \psi)$ in $X \times M$ such that

$$
\begin{aligned}
& \forall \theta \in X, \quad a(\omega, \theta)+b(\theta, \psi)=0, \\
& \forall \varphi \in M, \quad b(\omega, \varphi)=-\int_{\Omega} \boldsymbol{f} \cdot \operatorname{curl} \varphi \mathrm{d} \boldsymbol{x},
\end{aligned}
$$

where the bilinear forms $a(.,$.$) and b(.,$.$) are defined respectively on X \times X$ and $X \times M$ by

$$
a(\omega, \theta)=\int_{\Omega} \omega \theta \mathrm{d} \boldsymbol{x}, \quad b(\theta, \varphi)=\langle\Delta \theta, \varphi\rangle,
$$

the brackets $\langle\cdot, \cdot\rangle$ denoting the duality pairing between $H^{-1}(\Omega)$ and $H_{0}^{1}(\Omega)$.

Problem (2.4) is of saddle-point type, so its numerical analysis relies on the following arguments (Section 1 of Chapter III in [12]):

- the form $a(.,$.$) is continuous on L^{2}(\Omega) \times L^{2}(\Omega)$ and elliptic on the kernel

$$
V=\{\theta \in X ; \forall \varphi \in M, b(\theta, \varphi)=0\}=\{\theta \in X ; \Delta \theta=0 \text { in } \Omega\}
$$

- the form $b(.,$.$) is continuous on X \times M$ and satisfies the inf-sup condition, for a positive constant $\beta$ :

$$
\forall \varphi \in M, \quad \sup _{\theta \in X} \frac{b(\theta, \varphi)}{\|\theta\|_{X}} \geq \frac{\int_{\Omega}(\operatorname{curl} \varphi)^{2} \mathrm{~d} \boldsymbol{x}}{\|\varphi\|_{H^{1}(\Omega)}} \geq \beta|\varphi|_{H^{1}(\Omega)} .
$$

So, for any data $\boldsymbol{f}$ in $L^{2}(\Omega)^{2}$, it admits a unique solution which is the solution of problem (2.2).

The standard discretization of problem (2.2) is constructed by the Galerkin method applied to formulation (2.4), see [11] and Section 2 of Chapter III in [12], however a well-known algorithm for solving the corresponding discrete problem, due to Glowinski and Pironneau [13], relies on a decomposition of the vorticity $\omega$ as the sum of a harmonic part $\omega^{*}$ and a function $\omega^{0}$ with homogeneous boundary conditions. Since our discretization also relies on this decomposition, we present the corresponding problem, which reads:

$$
\begin{cases}-\Delta \omega^{0}=\operatorname{curl} f & \text { in } \Omega, \\ -\Delta \omega^{*}=0 & \text { in } \Omega, \\ -\Delta \psi=\omega^{0}+\omega^{*} & \text { in } \Omega, \\ \psi=\partial_{n} \psi=0 \quad \text { and } \quad \omega^{0}=0 & \text { on } \partial \Omega .\end{cases}
$$

It admits the following uncoupled variational formulation: find a function $\omega^{0}$ in $M$ such that

$$
\forall \eta \in M, \quad b\left(\omega^{0}, \eta\right)=-\int_{\Omega} \boldsymbol{f} . \operatorname{curl} \eta \mathrm{d} \boldsymbol{x},
$$

next: find a pair $\left(\omega^{*}, \psi\right)$ in $X \times M$ such that

$$
\begin{array}{ll}
\forall \theta \in X, & a\left(\omega^{*}, \theta\right)+b(\theta, \psi)=-a\left(\omega^{0}, \theta\right), \\
\forall \varphi \in M, & b\left(\omega^{*}, \varphi\right)=0 .
\end{array}
$$

Of course, by setting: $\omega=\omega^{0}+\omega^{*}$, we observe that problem (2.4) is completely equivalent to the system (2.6)(2.7). So, for any data $\boldsymbol{f}$ in $L^{2}(\Omega)^{2}$, this system has a unique solution. This solution satisfies

$$
\|\omega\|_{X}+|\psi|_{H^{1}(\Omega)} \leq c\|\boldsymbol{f}\|_{L^{2}(\Omega)^{2}} .
$$


Moreover, since the function $\psi$ is the solution of a biharmonic equation with homogeneous boundary data, it follows from the standard regularity results (Theorem 7.3.2.1 of [14]) that it also satisfies

$$
|\psi|_{W^{1,4}(\Omega)} \leq c\|\boldsymbol{f}\|_{L^{2}(\Omega)^{2}}
$$

Remark. In view of the discretization, it must be observed that for all functions $\theta$ and $\eta$ in $H_{0}^{1}(\Omega)$, the form $b(\cdot, \cdot)$ can be written equivalently

$$
b(\theta, \eta)=-\int_{\Omega} \operatorname{grad} \theta \cdot \operatorname{grad} \eta \mathrm{d} \boldsymbol{x},
$$

so that problem (2.6) is a standard Dirichlet problem with homogeneous boundary conditions.

\subsection{The discrete problem}

To discretize problem (2.4) or (2.6)-(2.7), we now assume that $\Omega$ is a polygon and we introduce a regular family $\left(\mathcal{T}_{h}\right)_{h}$ of triangulations of $\Omega$, in the sense that:

- each $\mathcal{T}_{h}$ is the set of a finite number of triangles such that $\bar{\Omega}=\cup_{K \in \mathcal{T}_{h}} K$, the intersection of two different triangles being either empty or a corner or an edge of both triangles;

- for all triangles $K$ in all $\mathcal{T}_{h}$, the ratio of the diameter $h_{K}$ of $K$ to the diameter of the inscribed circle in $K$ is smaller than a positive constant $\sigma$ independent of $h$.

We also denote by $\mathcal{E}_{h}$ the set of all edges of triangles in $\mathcal{T}_{h}$ that are not contained in $\partial \Omega$ and by $h_{e}$ the length of each edge $e$ in $\mathcal{E}_{h}$. As usual, $h$ is the largest of the diameters $h_{K}$ of triangles $K$ in $\mathcal{T}_{h}$ and, in all that follows, $c, c^{\prime}, c^{\prime \prime}$ stand for generic constants which are independent of $h$.

Next we define the discrete spaces:

$$
X_{h}=\left\{\theta_{h} \in \mathscr{C}^{0}(\bar{\Omega}) ; \forall K \in \mathcal{T}_{h}, \theta_{h \mid K} \in \mathbb{P}_{1}(K)\right\}, \quad M_{h}=X_{h} \cap H_{0}^{1}(\Omega),
$$

where $\mathbb{P}_{1}(K)$ denotes the space of affine functions on $K$. The space $X_{h}$ is spanned by the Lagrange polynomials associated with the corners of all triangles $K$ in $\mathcal{T}_{h}$.

Our modified discretization requires the introduction of new bilinear forms: for all functions $\omega_{h}$ and $\theta_{h}$ in $X_{h}$,

$$
a_{h}\left(\omega_{h}, \theta_{h}\right)=a\left(\omega_{h}, \theta_{h}\right)+\alpha_{h} A_{h}\left(\omega_{h}, \theta_{h}\right), \quad \text { with } \quad A_{h}\left(\omega_{h}, \theta_{h}\right)=\sum_{e \in \mathcal{E}_{h}} h_{e} \int_{e}\left[\partial_{n} \omega_{h}\right]\left[\partial_{n} \theta_{h}\right] \mathrm{d} \tau
$$

where $\left[\partial_{n} \cdot\right]$ denotes the jump of the normal derivative through the edge. Here, $\alpha_{h}$ stands for a positive constant which can depend on the triangulation $\mathcal{T}_{h}$, its choice is made precise later on. Thus, for any data $\boldsymbol{f}$ in $L^{2}(\Omega)^{2}$, the discrete problem consists in three steps:

- firstly, find a function $\omega_{h}^{0}$ in $M_{h}$ such that

$$
\forall \eta_{h} \in M_{h}, \quad b\left(\omega_{h}^{0}, \eta_{h}\right)=-\int_{\Omega} \boldsymbol{f} . \operatorname{curl} \eta_{h} \mathrm{~d} \boldsymbol{x}
$$

- secondly, find a pair $\left(\omega_{h}^{*}, \psi_{h}\right)$ in $X_{h} \times M_{h}$ such that

$$
\begin{aligned}
& \forall \theta_{h} \in X_{h}, \quad a_{h}\left(\omega_{h}^{*}, \theta_{h}\right)+b\left(\theta_{h}, \psi_{h}\right)=-a\left(\omega_{h}^{0}, \theta_{h}\right), \\
& \forall \varphi_{h} \in M_{h}, \quad b\left(\omega_{h}^{*}, \varphi_{h}\right)=0,
\end{aligned}
$$

- thirdly, set

$$
\omega_{h}=\omega_{h}^{0}+\omega_{h}^{*}
$$


Remark. Problem (2.11)-(2.13) coincides with the standard discretization when $\alpha_{h}$ is zero.

Remark. Another improved discretization was firstly proposed by Amara and El Dabaghi [2], where the stabilization term concerns the full vorticity $\omega_{h}$ : it is built via the Galerkin method applied to formulation (2.4), with a further term $A_{h}\left(\omega_{h}, \theta_{h}\right)$. We show later the advantages of the new formulation.

Problem (2.11) is the standard finite element discretization of problem (2.6), hence it is well-posed. Furthermore, since all norms on the finite-dimensional discrete space $X_{h}$ are equivalent, the form $a_{h}(\cdot, \cdot)$ is continuous on $X_{h} \times X_{h}$ and, since $A_{h}\left(\theta_{h}, \theta_{h}\right)$ is nonnegative for all $\theta_{h}$ in $X_{h}$, it is elliptic on $X_{h}$. Similarly, the continuity of the form $b(\cdot, \cdot)$ on $X \times M$ implies its continuity on $X_{h} \times M_{h}$ and the following inf-sup condition is easily derived for a positive constant $\beta$ independent of $h$ (by taking $\theta_{h}$ equal to $\varphi_{h}$ ):

$$
\forall \varphi_{h} \in M_{h}, \quad \sup _{\theta_{h} \in X_{h}} \frac{b\left(\theta_{h}, \varphi_{h}\right)}{\left\|\theta_{h}\right\|_{X}} \geq \beta\left|\varphi_{h}\right|_{H^{1}(\Omega)} .
$$

This leads to the following result.

Theorem 2.1. For any nonnegative constant $\alpha_{h}$ and for all functions $\boldsymbol{f}$ in $L^{2}(\Omega)^{2}$, problem (2.11)-(2.13) has a unique solution $\left(\omega_{h}, \psi_{h}\right)$ in $X_{h} \times M_{h}$.

Note as a conclusion that the modification we propose is not at all expensive: the decomposition (2.13) is most often used for solving the linear system resulting from the discrete problem. Moreover, when applied to piecewise affine functions, the form $A_{h}(\cdot, \cdot)$ is rather simple:

$$
A_{h}\left(\omega_{h}, \theta_{h}\right)=\sum_{e \in \mathcal{E}_{h}} h_{e}^{2}\left[\partial_{n} \omega_{h}\right]_{\mid e}\left[\partial_{n} \theta_{h}\right]_{\mid e} .
$$

\section{ERror estimates}

The aim of this section is to prove the convergence of $\left(\omega_{h}, \psi_{h}\right)$ towards $(\omega, \psi)$ in $L^{2}(\Omega) \times W^{1,4}(\Omega)$ with weak assumptions. To obtain this result, we derive some more general a priori estimates for the error between these solutions.

In all that follows, we need the semi-norm defined on $X_{h}$ by

$$
\left\|\theta_{h}\right\|_{*}=\left(A_{h}\left(\theta_{h}, \theta_{h}\right)\right)^{\frac{1}{2}}
$$

Indeed, we introduce the discrete kernel

$$
V_{h}=\left\{\theta_{h} \in X_{h} ; \forall \varphi_{h} \in M_{h}, b\left(\theta_{h}, \varphi_{h}\right)=0\right\},
$$

and we observe from the following lemma that this semi-norm can be used to construct a discrete analogue of the norm of $X$ on $V_{h}$. Another consequence is that the form $a_{h}(\cdot, \cdot)$ is elliptic on $V_{h}$ for the norm of $X$, with ellipticity constant inf $\left\{1, \alpha_{h} / c^{2}\right\}$, for the constant $c$ of this lemma.

Lemma 3.1. The following estimate holds for all functions $\theta_{h}$ in $V_{h}$

$$
\left\|\Delta \theta_{h}\right\|_{H^{-1}(\Omega)} \leq c\left\|\theta_{h}\right\|_{*} .
$$

Proof. We observe from (3.2) that, for all $\theta_{h}$ in $V_{h}$,

$$
\begin{aligned}
\left\|\Delta \theta_{h}\right\|_{H^{-1}(\Omega)} & =\sup _{\varphi \in H_{0}^{1}(\Omega)} \frac{\int_{\Omega} \operatorname{grad} \theta_{h} \cdot \operatorname{grad} \varphi \mathrm{d} \boldsymbol{x}}{|\varphi|_{H^{1}(\Omega)}} \\
& =\sup _{\varphi \in H_{0}^{1}(\Omega)} \inf _{\varphi_{h} \in M_{h}} \frac{\int_{\Omega} \operatorname{grad} \theta_{h} \cdot \operatorname{grad}\left(\varphi-\varphi_{h}\right) \mathrm{d} \boldsymbol{x}}{|\varphi|_{H^{1}(\Omega)}} .
\end{aligned}
$$


Integrating by parts implies

$$
\left\|\Delta \theta_{h}\right\|_{H^{-1}(\Omega)}=\sup _{\varphi \in H_{0}^{1}(\Omega)} \inf _{\varphi h \in M_{h}} \frac{\frac{1}{2} \sum_{e \in \mathcal{E}_{h}} \int_{e}\left[\partial_{n} \theta_{h}\right]\left(\varphi-\varphi_{h}\right) \mathrm{d} \tau}{|\varphi|_{H^{1}(\Omega)}},
$$

whence

$$
\left\|\Delta \theta_{h}\right\|_{H^{-1}(\Omega)} \leq \sup _{\varphi \in H_{0}^{1}(\Omega)} \inf _{\varphi h \in M_{h}} \frac{\frac{1}{2} \sum_{e \in \mathcal{E}_{h}}\left\|\left[\partial_{n} \theta_{h}\right]\right\|_{L^{2}(e)}\left\|\varphi-\varphi_{h}\right\|_{L^{2}(e)}}{|\varphi|_{H^{1}(\Omega)}} .
$$

So, choosing $\varphi_{h}$ equal to the image of $\varphi$ by a regularization operator with values in $M_{h}$, see for instance equation (4.10) in [4], and using a Cauchy-Schwarz inequality lead to the desired result.

The error estimates are established in several steps. The first one deals with the discretization (2.11) of the Laplace equation (2.6) for $\omega^{0}$ and is completely standard, see Theorem 13.1 in [9] for instance.

Proposition 3.2. The following estimate holds

$$
\left|\omega^{0}-\omega_{h}^{0}\right|_{H^{1}(\Omega)}=\inf _{\eta_{h} \in M_{h}}\left|\omega^{0}-\eta_{h}\right|_{H^{1}(\Omega)} .
$$

Next, we work with the saddle-point problem (2.12) and we once more need the discrete kernel $V_{h}$ introduced in (3.2).

Proposition 3.3. The following estimate holds

$$
\begin{aligned}
\left\|\omega^{*}-\omega_{h}^{*}\right\|_{L^{2}(\Omega)}+\sqrt{\alpha_{h}}\left\|\omega_{h}^{*}\right\|_{*} \leq & c\left(\left\|\omega^{0}-\omega_{h}^{0}\right\|_{L^{2}(\Omega)}+\inf _{\theta_{h} \in V_{h}}\left\{\left\|\omega^{*}-\theta_{h}\right\|_{L^{2}(\Omega)}+\sqrt{\alpha_{h}}\left\|\theta_{h}\right\|_{*}\right\}\right. \\
& \left.+\sup _{\tau_{h} \in V_{h}} \inf _{\varphi_{h} \in M_{h}} \frac{b\left(\tau_{h}, \psi-\varphi_{h}\right)}{\left\|\tau_{h}\right\|_{L^{2}(\Omega)}+\sqrt{\alpha_{h}}\left\|\tau_{h}\right\|_{*}}\right) .
\end{aligned}
$$

Proof. Let $\theta_{h}$ be any function in $V_{h}$. Thus, the function $\tau_{h}=\omega_{h}^{*}-\theta_{h}$ belongs to $V_{h}$ and satisfies

$$
\left\|\tau_{h}\right\|_{L^{2}(\Omega)}^{2}+\alpha_{h}\left\|\tau_{h}\right\|_{*}^{2}=\int_{\Omega}\left(\omega_{h}^{*}-\omega^{*}\right) \tau_{h} \mathrm{~d} \boldsymbol{x}+\alpha_{h} A_{h}\left(\omega_{h}^{*}, \tau_{h}\right)+\int_{\Omega}\left(\omega^{*}-\theta_{h}\right) \tau_{h} \mathrm{~d} \boldsymbol{x}-\alpha_{h} A_{h}\left(\theta_{h}, \tau_{h}\right) .
$$

Using now problems (2.7) and (2.12) together with the definition (3.2) of $V_{h}$, gives, for any $\varphi_{h}$ in $M_{h}$,

$$
\left\|\tau_{h}\right\|_{L^{2}(\Omega)}^{2}+\alpha_{h}\left\|\tau_{h}\right\|_{*}^{2}=b\left(\tau_{h}, \psi-\varphi_{h}\right)+\int_{\Omega}\left(\omega^{*}-\theta_{h}\right) \tau_{h} \mathrm{~d} \boldsymbol{x}-\alpha_{h} A_{h}\left(\theta_{h}, \tau_{h}\right)+\int_{\Omega}\left(\omega^{0}-\omega_{h}^{0}\right) \tau_{h} \mathrm{~d} \boldsymbol{x} .
$$

This yields

$$
\begin{aligned}
\left\|\omega_{h}^{*}-\theta_{h}\right\|_{L^{2}(\Omega)}+\sqrt{\alpha_{h}}\left\|\omega_{h}^{*}-\theta_{h}\right\|_{*} \leq c & \frac{\left|b\left(\tau_{h}, \psi-\varphi_{h}\right)\right|}{\left\|\tau_{h}\right\|_{L^{2}(\Omega)}+\sqrt{\alpha_{h}}\left\|\tau_{h}\right\|_{*}}+\left\|\omega^{*}-\theta_{h}\right\|_{L^{2}(\Omega)}+\sqrt{\alpha_{h}}\left\|\theta_{h}\right\|_{*} \\
& \left.+\left\|\omega^{0}-\omega_{h}^{0}\right\|_{L^{2}(\Omega)}\right) .
\end{aligned}
$$

The desired result follows by using two triangular inequalities.

As standard in the numerical analysis of this discretization ((A.25) of Chapter III in [12]), we now introduce the projection operator $P_{h}$, with values in $X_{h}$, defined for all functions $\mu$ in $W^{1,1}(\Omega)$ by

$$
\forall \theta_{h} \in X_{h}, \quad \int_{\Omega} \operatorname{grad}\left(\mu-P_{h} \mu\right) \cdot \operatorname{grad} \theta_{h} \mathrm{~d} \boldsymbol{x}=0 \quad \text { and } \quad \int_{\Omega} P_{h} \mu \mathrm{d} \boldsymbol{x}=\int_{\Omega} \mu \mathrm{d} \boldsymbol{x} .
$$


Proposition 3.4. The following estimate holds

$$
\begin{aligned}
\left|\psi-\psi_{h}\right|_{W^{1,4}(\Omega)} \leq & c\left(\left|\psi-P_{h} \psi\right|_{W^{1,4}(\Omega)}+\left\|\omega-\omega_{h}\right\|_{L^{2}(\Omega)} \sup _{\mu \in W^{1, \frac{4}{3}}(\Omega)} \frac{\left\|\mu-P_{h} \mu\right\|_{L^{2}(\Omega)}}{|\mu|_{W^{1, \frac{4}{3}}(\Omega)}}\right. \\
& \left.+\alpha_{h}\left\|\omega_{h}^{*}\right\|_{*} \sup _{\mu \in W^{1, \frac{4}{3}(\Omega)}} \frac{\left\|P_{h} \mu\right\|_{*}}{|\mu|_{W^{1, \frac{4}{3}}(\Omega)}}+h\left|\omega^{0}-\omega_{h}^{0}\right|_{H^{1}(\Omega)}+h\left\|\omega_{h}^{*}\right\|_{*}\right) .
\end{aligned}
$$

Proof. We have

$$
\left|\psi-\psi_{h}\right|_{W^{1,4}(\Omega)}=\sup _{\boldsymbol{g} \in L^{\frac{4}{3}}(\Omega)^{2}} \frac{\int_{\Omega} \boldsymbol{g} \cdot \operatorname{grad}\left(\psi-\psi_{h}\right) \mathrm{d} \boldsymbol{x}}{\|\boldsymbol{g}\|_{L^{\frac{4}{3}}(\Omega)^{2}}} .
$$

So, let $\boldsymbol{g}$ be any function in $L^{\frac{4}{3}}(\Omega)^{2}$. Then, div $\boldsymbol{g}$ belongs to $W^{-1, \frac{4}{3}}(\Omega)$ and we observe from Theorem 7.3.2.1 of [14] that the solution $\chi$ of the problem

$$
\begin{cases}\Delta^{2} \chi=\operatorname{div} \boldsymbol{g} & \text { in } \Omega, \\ \chi=\partial_{n} \chi=0 & \text { on } \partial \Omega,\end{cases}
$$

belongs to $W^{3, \frac{4}{3}}(\Omega)$ and satisfies

$$
\|\chi\|_{W^{3, \frac{4}{3}(\Omega)}} \leq c\|\boldsymbol{g}\|_{L^{\frac{4}{3}(\Omega)^{2}}} .
$$

Then, the function $\mu=-\Delta \chi$ belongs to $W^{1, \frac{4}{3}}(\Omega)$ and satisfies: $-\Delta \mu=\operatorname{div} \boldsymbol{g}$. So, integrating twice by parts, we have

$$
\int_{\Omega} \boldsymbol{g} \cdot \operatorname{grad}\left(\psi-\psi_{h}\right) \mathrm{d} \boldsymbol{x}=-\int_{\Omega} \operatorname{grad} \mu \cdot \operatorname{grad}\left(\psi-\psi_{h}\right) \mathrm{d} \boldsymbol{x} .
$$

By using problems (2.7) and (2.12) and the definition (3.6) of $P_{h}$, we obtain

$$
\begin{aligned}
\int_{\Omega} \boldsymbol{g} \cdot \operatorname{grad}\left(\psi-\psi_{h}\right) \mathrm{d} \boldsymbol{x}= & \int_{\Omega} \operatorname{grad}\left(P_{h} \mu-\mu\right) \cdot \operatorname{grad}\left(\psi-\psi_{h}\right) \mathrm{d} \boldsymbol{x}-\int_{\Omega} \operatorname{grad} P_{h} \mu \cdot \operatorname{grad}\left(\psi-\psi_{h}\right) \mathrm{d} \boldsymbol{x} \\
= & \int_{\Omega} \operatorname{grad}\left(P_{h} \mu-\mu\right) \cdot \operatorname{grad}\left(\psi-P_{h} \psi\right) \mathrm{d} \boldsymbol{x}-a\left(\omega-\omega_{h}, P_{h} \mu\right)+\alpha_{h} A_{h}\left(\omega_{h}^{*}, P_{h} \mu\right) \\
= & -\int_{\Omega} \operatorname{grad} \mu \cdot \operatorname{grad}\left(\psi-P_{h} \psi\right) \mathrm{d} \boldsymbol{x}+a\left(\omega-\omega_{h}, \mu-P_{h} \mu\right) \\
& +\alpha_{h} A_{h}\left(\omega_{h}^{*}, P_{h} \mu\right)+b\left(\omega-\omega_{h}, \chi\right) .
\end{aligned}
$$


So, it remains to bound each of the four terms in the right-hand side. The first three ones are easy:

$$
\begin{aligned}
\left|\int_{\Omega} \operatorname{grad} \mu \cdot \operatorname{grad}\left(\psi-P_{h} \psi\right) \mathrm{d} \boldsymbol{x}\right| & \leq|\mu|_{W^{1, \frac{4}{3}}(\Omega)}\left|\psi-P_{h} \psi\right|_{W^{1,4}(\Omega)} \\
& \leq c\left|\psi-P_{h} \psi\right|_{W^{1,4}(\Omega)}\|\boldsymbol{g}\|_{L^{\frac{4}{3}}(\Omega)^{2}}, \\
\left|a\left(\omega-\omega_{h}, \mu-P_{h} \mu\right)\right| & \leq c\left\|\omega-\omega_{h}\right\|_{L^{2}(\Omega)} \frac{\left\|\mu-P_{h} \mu\right\|_{L^{2}(\Omega)}}{|\mu|_{W^{1, \frac{4}{3}}(\Omega)}}\|\boldsymbol{g}\|_{L^{\frac{4}{3}}(\Omega)^{2}}, \\
\left|A_{h}\left(\omega_{h}^{*}, P_{h} \mu\right)\right| & \leq\left\|\omega_{h}^{*}\right\|_{*}\left\|P_{h} \mu\right\|_{*} \leq c\left\|\omega_{h}^{*}\right\|_{*} \frac{\left\|P_{h} \mu\right\|_{*}}{|\mu|_{W^{1, \frac{4}{3}}(\Omega)}}\|\boldsymbol{g}\|_{L^{\frac{4}{3}}(\Omega)^{2}} .
\end{aligned}
$$

Concerning the fourth one, we have for all $\chi_{h}$ in $M_{h}$,

$$
b\left(\omega-\omega_{h}, \chi\right)=b\left(\omega-\omega_{h}, \chi-\chi_{h}\right)=\left\langle\Delta\left(\omega^{0}-\omega_{h}^{0}\right), \chi-\chi_{h}\right\rangle-\left\langle\Delta \omega_{h}^{*}, \chi-\chi_{h}\right\rangle,
$$

whence

$$
\left|b\left(\omega-\omega_{h}, \chi\right)\right| \leq\left(\left|\omega^{0}-\omega_{h}^{0}\right|_{H^{1}(\Omega)}+\left\|\Delta \omega_{h}^{*}\right\|_{H^{-1}(\Omega)}\right)\left|\chi-\chi_{h}\right|_{H^{1}(\Omega)} .
$$

So, noting that $W^{3, \frac{4}{3}}(\Omega)$ is included in $H^{2}(\Omega)$ and taking $\chi_{h}$ equal to the standard Lagrange interpolate of $\chi$, we derive from (3.9) that

$$
\left|b\left(\omega-\omega_{h}, \chi\right)\right| \leq c h\left(\left|\omega^{0}-\omega_{h}^{0}\right|_{H^{1}(\Omega)}+\left\|\Delta \omega_{h}^{*}\right\|_{H^{-1}(\Omega)}\right)\|\boldsymbol{g}\|_{L^{\frac{4}{3}}(\Omega)^{2}} .
$$

Finally, applying Lemma 3.1 to $\omega_{h}^{*}$ gives

$$
\left|b\left(\omega-\omega_{h}, \chi\right)\right| \leq c h\left(\left|\omega^{0}-\omega_{h}^{0}\right|_{H^{1}(\Omega)}+\left\|\omega_{h}^{*}\right\|_{*}\right)\|\boldsymbol{g}\|_{L^{\frac{4}{3}}(\Omega)^{2}} .
$$

And combining all these estimates leads to the desired result.

The next step consists in evaluating the unusual terms that appear in the right-hand side of estimate (3.7), namely

$$
\sup _{\mu \in W^{1, \frac{4}{3}}(\Omega)} \frac{\left\|\mu-P_{h} \mu\right\|_{L^{2}(\Omega)}}{|\mu|_{W^{1, \frac{4}{3}}(\Omega)}} \quad \text { and } \quad \sup _{\mu \in W^{1, \frac{4}{3}(\Omega)}} \frac{\left\|P_{h} \mu\right\|_{*}}{|\mu|_{W^{1, \frac{4}{3}}(\Omega)}} .
$$

We need some further notation for that.

Notation. We denote by $h_{\min }$ the smallest of the diameters $h_{K}, K \in \mathcal{T}_{h}$. Let $\boldsymbol{a}_{i}, 1 \leq i \leq N$, be the nonconvex corners of $\Omega$, i.e. the corners in which the aperture of the angle is larger than $\pi$. We introduce a fixed neighbourhood $U_{i}$ of each corner $\boldsymbol{a}_{i}$, and we assume that

$$
U_{i} \cap U_{j}=\emptyset, \quad 1 \leq i<j \leq N .
$$

In what follows, $h_{i}$ stands for the largest of the diameters $h_{K}$ of triangles $K$ of $\mathcal{T}_{h}$ that intersect $U_{i}$.

Lemma 3.5. There exist real numbers $\alpha_{i}, 1 \leq i \leq N$, satisfying: $1 / 2<\alpha_{i}<1$ and such that:

$$
\sup _{\mu \in W^{1, \frac{4}{3}}(\Omega)} \frac{\left\|\mu-P_{h} \mu\right\|_{L^{2}(\Omega)}}{|\mu|_{W^{1, \frac{4}{3}}(\Omega)}} \leq c h_{\min }^{-\frac{1}{2}}\left(h+\sum_{i=1}^{N} h_{i}^{\alpha_{i}}\right) .
$$


Proof. Let $\mu$ be any function in $W^{1, \frac{4}{3}}(\Omega)$. Evaluating the $L^{2}(\Omega)$ norm of $\mu-P_{h} \mu$ relies on a duality argument. So, we consider the solution $\xi$ in $H^{1}(\Omega)$ with null integral of the Neumann problem

$$
\begin{cases}-\Delta \xi=\mu-P_{h} \mu & \text { in } \Omega, \\ \partial_{n} \xi=0 & \text { on } \partial \Omega .\end{cases}
$$

Note that the function $\xi$ belongs to $W^{1,4}(\Omega)$. We have

$$
\begin{aligned}
\left\|\mu-P_{h} \mu\right\|_{L^{2}(\Omega)}^{2} & =\int_{\Omega} \operatorname{grad} \xi \cdot \operatorname{grad}\left(\mu-P_{h} \mu\right) \mathrm{d} \boldsymbol{x}=\int_{\Omega} \operatorname{grad}\left(\xi-P_{h} \xi\right) \cdot \operatorname{grad} \mu \mathrm{d} \boldsymbol{x} \\
& \leq\left|\xi-P_{h} \xi\right|_{W^{1,4}(\Omega)}|\mu|_{W^{1, \frac{4}{3}}(\Omega)}
\end{aligned}
$$

so that

$$
\frac{\left\|\mu-P_{h} \mu\right\|_{L^{2}(\Omega)}}{|\mu|_{W^{1, \frac{4}{3}}(\Omega)}} \leq \frac{\left|\xi-P_{h} \xi\right|_{W^{1,4}(\Omega)}}{\|\Delta \xi\|_{L^{2}(\Omega)}} .
$$

Next, for any $\xi_{h}$ in $X_{h}$, from the local inverse inequality

$$
\left|\xi-P_{h} \xi\right|_{W^{1,4}(K)} \leq\left|\xi-\xi_{h}\right|_{W^{1,4}(K)}+c h_{K}^{-\frac{1}{2}}\left(\left|\xi-P_{h} \xi\right|_{H^{1}(K)}+\left|\xi-\xi_{h}\right|_{H^{1}(K)}\right),
$$

we derive by Jensen's inequality

$$
\left|\xi-P_{h} \xi\right|_{W^{1,4}(\Omega)} \leq\left|\xi-\xi_{h}\right|_{W^{1,4}(\Omega)}+c h_{\min }^{-\frac{1}{2}}\left(\left|\xi-P_{h} \xi\right|_{H^{1}(\Omega)}+\left|\xi-\xi_{h}\right|_{H^{1}(\Omega)}\right),
$$

whence, from the definition of $P_{h}$,

$$
\left|\xi-P_{h} \xi\right|_{W^{1,4}(\Omega)} \leq\left|\xi-\xi_{h}\right|_{W^{1,4}(\Omega)}+c h_{\min }^{-\frac{1}{2}}\left|\xi-\xi_{h}\right|_{H^{1}(\Omega)} .
$$

To evaluate the terms in the right-hand side, we recall from Chapter 4 of [14] that the solution $\xi$ of (3.11) can be written as the sum of a regular part $\xi_{r}$ in $H^{2}(\Omega)$ and of a linear combination $\sum_{i=1}^{N} \lambda_{i} S_{i}$, where the $\lambda_{i}$ are real constants and the $S_{i}$ are singular functions with support in $U_{i}$. Moreover, the following estimate holds:

$$
\left\|\xi_{r}\right\|_{H^{2}(\Omega)}+\sum_{i=1}^{N}\left|\lambda_{i}\right| \leq c\|\Delta \xi\|_{L^{2}(\Omega)},
$$

and the $S_{i}$ belong to $H^{1+\alpha_{i}}\left(U_{i}\right)$ for real numbers $\alpha_{i}$ satisfying the conditions of the lemma (more precisely, each $\alpha_{i}$ is smaller than $\pi$ divided by the aperture of the angle in $\boldsymbol{a}_{i}$ ). So, we now choose $\xi_{h}$ equal to the Lagrange interpolate of $\xi$, we observe that it can be written as the sum $\xi_{r h}+\sum_{i=1}^{N} \lambda_{i} S_{i h}$, where $\xi_{r h}$ and $S_{i h}$ are the Lagrange interpolates of $\xi_{j}$ and the $S_{i}$ respectively. Since the support of each $S_{i h}$ is contained in the union of triangles $K$ of $\mathcal{T}_{h}$ that intersect $U_{i}$, we have the estimates

$$
\left|\xi_{r}-\xi_{r h}\right|_{H^{1}(\Omega)} \leq c h\left\|\xi_{r}\right\|_{H^{2}(\Omega)} \quad \text { and } \quad\left|S_{i}-S_{i h}\right|_{H^{1}(\Omega)} \leq c h_{i}^{\alpha_{i}}\left\|S_{i}\right\|_{H^{1+\alpha_{i}(\Omega)}},
$$

and also, due to the embeddings of $H^{2}(\Omega)$ and $H^{1+\alpha_{i}}(\Omega)$ into $W^{\frac{3}{2}, 4}(\Omega)$ and $W^{\frac{1}{2}+\alpha_{i}, 4}(\Omega)$ respectively,

$$
\left|\xi_{r}-\xi_{r h}\right|_{W^{1,4}(\Omega)} \leq c h^{\frac{1}{2}}\left\|\xi_{r}\right\|_{H^{2}(\Omega)} \quad \text { and } \quad\left|S_{i}-S_{i h}\right|_{W^{1,4}(\Omega)} \leq c h_{i}^{\alpha_{i}-\frac{1}{2}}\left\|S_{i}\right\|_{H^{1+\alpha_{i}}(\Omega)} .
$$

We conclude by combining all these estimates and noting that $h \geq h_{\min }$ and $h_{i} \geq h_{\min }$. 
Lemma 3.6. The following estimate holds

$$
\sup _{\mu \in W^{1, \frac{4}{3}}(\Omega)} \frac{\left\|P_{h} \mu\right\|_{*}}{|\mu|_{W^{1, \frac{4}{3}}(\Omega)}} \leq c h_{\min }^{-\frac{1}{2}} .
$$

Proof. Let $\mu$ be any function in $W^{1, \frac{4}{3}}(\Omega)$. We introduce the function $\Phi$ in $H_{0}^{1}(\Omega)$ such that its restriction to each $K$ in $\mathcal{T}_{h}$ is a polynomial of degree $\leq 2$, which vanishes at all corners of all $K$ in $\mathcal{T}_{h}$ and is equal to $\frac{3}{2} h_{e}\left[\partial_{n} P_{h} \mu\right]_{\mid e}$ at the midpoint of each edge $e$ in $\mathcal{E}_{h}$. Equivalently, if $\varphi_{e}$ denotes the Lagrange polynomial associated with the midpoint of $e$ (which vanishes in all endpoints and midpoints of all edges $\neq e$ of elements of $\mathcal{T}_{h}$ ), the function $\Phi$ is given by

$$
\Phi=\frac{3}{2} \sum_{e \in \mathcal{E}_{h}} h_{e}\left[\partial_{n} P_{h} \mu\right]_{\mid e} \varphi_{e}
$$

We observe that, for all $\theta_{h}$ in $X_{h}$,

$$
A_{h}\left(P_{h} \mu, \theta_{h}\right)=\sum_{e \in \mathcal{E}_{h}} \int_{e} \Phi(\tau)\left[\partial_{n} \theta_{h}\right] \mathrm{d} \tau=b\left(\theta_{h}, \Phi\right),
$$

from which we deduce

$$
\left\|P_{h} \mu\right\|_{*}^{2}=b\left(P_{h} \mu, \Phi\right) \leq\left|P_{h} \Phi\right|_{W^{1,4}(\Omega)}|\mu|_{W^{1, \frac{4}{3}(\Omega)}} .
$$

Using an inverse inequality together with Jensen's inequality gives

$$
\left\|P_{h} \mu\right\|_{*}^{2} \leq c h_{\min }^{-\frac{1}{2}}\left|P_{h} \Phi\right|_{H^{1}(\Omega)}|\mu|_{W^{1, \frac{4}{3}}(\Omega)} \leq c h_{\min }^{-\frac{1}{2}}|\Phi|_{H^{1}(\Omega)}|\mu|_{W^{1, \frac{4}{3}}(\Omega)} .
$$

So it remains to estimate $|\Phi|_{H^{1}(\Omega)}$. We note that $\left|\varphi_{e}\right|_{H^{1}(\Omega)}$ is bounded independently of $h$ and that the support of $\varphi_{e}$ is made of the two triangles which share the edge $e$, so that it is orthogonal to all other functions $\varphi_{e^{\prime}}$ but at most four. Due to (2.15), we obtain

$$
|\Phi|_{H^{1}(\Omega)} \leq c\left(\sum_{e \in \mathcal{E}_{h}}\left(\frac{3}{2} h_{e}\left[\partial_{n} P_{h} \mu\right]_{\mid e}\right)^{2}\right)^{\frac{1}{2}} \leq c^{\prime}\left\|P_{h} \mu\right\|_{*}
$$

This ends the proof.

Remark. Using exactly the same arguments yields that, for all functions $\theta_{h}$ in $X_{h}$,

$$
\left\|\theta_{h}\right\|_{*} \leq c\left\|\Delta \theta_{h}\right\|_{H^{-1}(\Omega)} .
$$

Indeed, choosing $\Phi$ equal to

$$
\Phi=\frac{3}{2} \sum_{e \in \mathcal{E}_{h}} h_{e}\left[\partial_{n} \theta_{h}\right]_{\mid e} \varphi_{e}
$$

we have

$$
\left\|\theta_{h}\right\|_{*}^{2}=b\left(\theta_{h}, \Phi\right) \leq\left\|\Delta \theta_{h}\right\|_{H^{-1}(\Omega)}|\Phi|_{H^{1}(\Omega)} \quad \text { and } \quad|\Phi|_{H^{1}(\Omega)} \leq c\left\|\theta_{h}\right\|_{*} .
$$


So, combining this result with Lemma 3.1, we derive that the norm

$$
\left(a\left(\theta_{h}, \theta_{h}\right)+A_{h}\left(\theta_{h}, \theta_{h}\right)\right)^{\frac{1}{2}}
$$

is equivalent to the norm of $X$ on $V_{h}$, with equivalence constants independent of $h$. As a consequence, the discrete problem (2.12) satisfies the properties of uniform ellipticity and uniform inf-sup condition when $\alpha_{h}$ is a constant independent of $h$, and this leads to the simpler estimate

$$
\left\|\omega^{*}-\omega_{h}^{*}\right\|_{X}+\left|\psi-\psi_{h}\right|_{H^{1}(\Omega)} \leq c\left(\inf _{\theta_{h} \in X_{h}}\left\|\omega^{*}-\theta_{h}\right\|_{X}+\inf _{\varphi_{h} \in M_{h}}\left|\psi-\varphi_{h}\right|_{H^{1}(\Omega)}+\left\|\omega^{0}-\omega_{h}^{0}\right\|_{L^{2}(\Omega)}\right) .
$$

This is sufficient to prove the convergence of $\omega_{h}^{*}$ towards $\omega^{*}$ in $X$ but not the convergence of $\psi_{h}$ towards $\psi$ in $W^{1,4}(\Omega)$.

We are now in a position to bound the right-hand sides of (3.4), (3.5) and (3.7). Firstly, we observe from (3.4) that, if the data $\boldsymbol{f}$ belong to $L^{2}(\Omega)^{2}$, the solution $\omega_{h}^{0}$ converges towards the solution $\omega^{0}$ of problem $(2.6)$ in $H^{1}(\Omega)$. Moreover applying the duality argument of Aubin-Nitsche leads to the estimate

$$
\left\|\omega^{0}-\omega_{h}^{0}\right\|_{L^{2}(\Omega)} \leq c\left(h+\sum_{i=1}^{N} h_{i}^{\alpha_{i}}\right)\left|\omega^{0}-\omega_{h}^{0}\right|_{H^{1}(\Omega)}
$$

Concerning estimate (3.5), we observe that the solution $\left(\omega^{*}, \psi\right)$ of problem $(2.7)$ belongs to $W^{1, \frac{4}{3}}(\Omega) \times H^{2}(\Omega)$. By noting that $P_{h} \omega^{*}$ belongs to $V_{h}$, applying Lemmas 3.5 and 3.6 to $\omega^{*}$ yields

$$
\left\|\omega^{*}-P_{h} \omega^{*}\right\|_{L^{2}(\Omega)}+\sqrt{\alpha_{h}}\left\|P_{h} \omega^{*}\right\|_{*} \leq c h_{\min }^{-\frac{1}{2}}\left(h+\sum_{i=1}^{N} h_{i}^{\alpha_{i}}+\sqrt{\alpha_{h}}\right)\left\|\omega^{*}\right\|_{W^{1, \frac{4}{3}}(\Omega)} .
$$

Finally, we use Lemma 3.1 to derive that, for all $\tau_{h}$ in $V_{h}$,

$$
\begin{aligned}
\inf _{\varphi_{h} \in M_{h}} \frac{b\left(\tau_{h}, \psi-\varphi_{h}\right)}{\left\|\tau_{h}\right\|_{L^{2}(\Omega)}+\sqrt{\alpha_{h}}\left\|\tau_{h}\right\|_{*}} & \leq \inf _{\varphi_{h} \in M_{h}} \frac{\left\|\Delta \tau_{h}\right\|_{H^{-1}(\Omega)}\left|\psi-\varphi_{h}\right|_{H^{1}(\Omega)}}{\left\|\tau_{h}\right\|_{L^{2}(\Omega)}+\sqrt{\alpha_{h}}\left\|\tau_{h}\right\|_{*}} \\
& \leq \frac{1}{\sqrt{\alpha_{h}}} \inf _{\varphi_{h} \in M_{h}}\left|\psi-\varphi_{h}\right|_{H^{1}(\Omega)},
\end{aligned}
$$

and we can take $\varphi_{h}$ equal to the Lagrange interpolate of $\psi$ to conclude. So we have estimated all the terms of (3.5). Next, in estimate (3.7), we bound the first term in the right-hand side thanks to (3.13) and we make use of Lemmas 3.5 and 3.6 once more. Finally, the quantity $\sqrt{\alpha_{h}}\left\|\Delta\left(\omega-\omega_{h}\right)\right\|_{H^{-1}(\Omega)}$ is bounded by the sum of $\sqrt{\alpha_{h}}\left\|\Delta\left(\omega^{0}-\omega_{h}^{0}\right)\right\|_{H^{-1}(\Omega)}$ and of $\sqrt{\alpha_{h}}\left\|\Delta\left(\omega^{*}-\omega_{h}^{*}\right)\right\|_{H^{-1}(\Omega)}=\sqrt{\alpha_{h}}\left\|\Delta \omega_{h}^{*}\right\|_{H^{-1}(\Omega)}$, this last term being estimated thanks to Lemma 3.1.

Theorem 3.7. The following error estimate holds between the solution $(\omega, \psi)$ of problem (2.4) and the solution $\left(\omega_{h}, \psi_{h}\right)$ of problem $(2.11)-(2.13)$ :

$$
\left\|\omega-\omega_{h}\right\|_{L^{2}(\Omega)}+\sqrt{\alpha_{h}}\left\|\Delta\left(\omega-\omega_{h}\right)\right\|_{H^{-1}(\Omega)}+\left|\psi-\psi_{h}\right|_{W^{1,4}(\Omega)} \leq c E_{h} \sup \left\{E_{h}, 1\right\}\|\boldsymbol{f}\|_{L^{2}(\Omega)^{2}},
$$

where $E_{h}$ denotes the quantity

$$
E_{h}=h_{\min }^{-\frac{1}{2}}\left(h+\sum_{i=1}^{N} h_{i}^{\alpha_{i}}+\sqrt{\alpha_{h}}\right)+\frac{h}{\sqrt{\alpha_{h}}} .
$$


If the following assumption holds:

$$
h_{i} \leq c h_{\min }, \quad 1 \leq i \leq N
$$

and if $\alpha_{h}$ is chosen such that

$$
\lim _{h \rightarrow 0} h_{\min }^{-1} \alpha_{h}=0 \quad \text { and } \quad \lim _{h \rightarrow 0} h^{2} \alpha_{h}^{-1}=0
$$

the following convergence property holds:

$$
\lim _{h \rightarrow 0}\left\|\omega-\omega_{h}\right\|_{L^{2}(\Omega)}+\sqrt{\alpha_{h}}\left\|\Delta\left(\omega-\omega_{h}\right)\right\|_{H^{-1}(\Omega)}+\left|\psi-\psi_{h}\right|_{W^{1,4}(\Omega)}=0 .
$$

Note that, in the general case of a nonconvex geometry and for the standard discretization $\left(\alpha_{h}=0\right)$, the convergence of $\omega-\omega_{h}$ in $L^{2}(\Omega)$ is not proven and seems not to hold from numerical experiments. This convergence is proven for the modified discretization proposed in [2], however the convergence of $\psi-\psi_{h}$ in $W^{1,4}(\Omega)$ is established neither for the standard discretization nor for the modified one of [2].

Remark. Assumption (3.20) only concerns the triangulation $\mathcal{T}_{h}$, note that it is much less restrictive than the uniform regularity: using triangles of minimal size in a neighbourhood of the nonconvex corners seems reasonable. Assumption (3.21) also implies the condition $\lim _{h \rightarrow 0} h_{\min }^{-1} h^{2}=0$, however the inequality $h_{\text {min }}>$ $h^{2-\varepsilon}$ for a positive real number $\varepsilon$ is satisfied in most practical situations.

Remark. When the domain $\Omega$ is convex $(N=0)$ and the family of triangulations $\left(\mathcal{T}_{h}\right)_{h}$ is uniformly regular $\left(h_{\min } \geq c h\right)$, the following estimate is known in the case $\alpha_{h}=0$ of the standard discretization

$$
\left|\psi-\psi_{h}\right|_{W^{1,4}(\Omega)} \leq c h^{\frac{1}{2}}\|\boldsymbol{f}\|_{L^{2}(\Omega)^{2}}
$$

Its proof relies on an argument due to Scholz [15], see for instance Theorem 3.1 of Chapter II in [12]. And this estimate is still valid for our modified discretization with $\alpha_{h}=h$, it can be derived by replacing (3.17) by an improved estimate relying on the fact that $\omega^{*}$ belongs to $H^{1}(\Omega)$.

We conclude with a stability result which is needed for the extension to the Navier-Stokes equations.

Corollary 3.8. Assume that conditions (3.20) and (3.21) are satisfied. For all functions $\boldsymbol{f}^{\text {in }} L^{\frac{4}{3}}(\Omega)^{2}$, problem (2.11)-(2.13) has a unique solution $\left(\omega_{h}, \psi_{h}\right)$ in $X_{h} \times M_{h}$. Moreover, this solution satisfies

$$
\left\|\omega_{h}\right\|_{L^{2}(\Omega)}+\left|\psi_{h}\right|_{W^{1,4}(\Omega)} \leq c\|\boldsymbol{f}\|_{L^{\frac{4}{3}(\Omega)^{2}}} .
$$

Proof. The arguments are very similar to the previous ones. The existence for $\boldsymbol{f}$ in $L^{\frac{4}{3}}(\Omega)^{2}$ comes from the continuity of the right-hand side, since $M_{h}$ is included in $W^{1,4}(\Omega)$. Next, choosing $\eta_{h}$ equal to $\omega_{h}^{0}$ in $(2.11)$ yields, thanks to an inverse inequality in the right-hand side,

$$
\left\|\omega_{h}^{0}\right\|_{H^{1}(\Omega)} \leq h_{\min }^{-\frac{1}{2}}\|\boldsymbol{f}\|_{L^{\frac{4}{3}}(\Omega)^{2}}
$$

Taking $\theta_{h}$ equal to $\omega_{h}$ in the first line of (2.12) and combining it with the second line yield

$$
\left\|\omega_{h}\right\|_{L^{2}(\Omega)}^{2}+\alpha_{h}\left\|\omega_{h}^{*}\right\|_{*}^{2}=-b\left(\omega_{h}, \psi_{h}\right)-\alpha_{h} A_{h}\left(\omega_{h}^{*}, \omega_{h}^{0}\right)=-b\left(\omega_{h}^{0}, \psi_{h}\right)-\alpha_{h} A_{h}\left(\omega_{h}^{*}, \omega_{h}^{0}\right) .
$$

Using a Cauchy-Schwarz inequality in the last term leads to

$$
\left\|\omega_{h}\right\|_{L^{2}(\Omega)}^{2}+\frac{\alpha_{h}}{2}\left\|\omega_{h}^{*}\right\|_{*}^{2} \leq-b\left(\omega_{h}^{0}, \psi_{h}\right)+\frac{\alpha_{h}}{2} A_{h}\left(\omega_{h}^{0}, \omega_{h}^{0}\right) .
$$


Combining the inverse inequality on each edge $e$ of a triangle $K$

$$
\left\|\partial_{n} \omega_{h}^{0}\right\|_{L^{2}(e)} \leq c h_{e}^{-\frac{1}{2}}\left\|\omega_{h}^{0}\right\|_{H^{1}(K)},
$$

with the previous estimate on $\omega_{h}^{0}$, we deduce from (2.11) that

$$
\left\|\omega_{h}\right\|_{L^{2}(\Omega)}^{2}+\frac{\alpha_{h}}{2}\left\|\omega_{h}^{*}\right\|_{*}^{2} \leq \int_{\Omega} \boldsymbol{f} \cdot \operatorname{curl} \psi_{h} \mathrm{~d} \boldsymbol{x}+c h_{\min }^{-1} \alpha_{h}\|\boldsymbol{f}\|_{L^{\frac{4}{3}(\Omega)^{2}}}^{2} .
$$

As in the proof of Proposition 3.4, problem

$$
\begin{cases}\Delta^{2} \chi=\operatorname{curl} f & \text { in } \Omega, \\ \chi=\partial_{n} \chi=0 & \text { on } \partial \Omega,\end{cases}
$$

has a unique solution $\chi$ in $W^{3, \frac{4}{3}}(\Omega)$, so that the function $\mu=-\Delta \chi$ belongs to $W^{1, \frac{4}{3}}(\Omega)$. This gives

$$
\int_{\Omega} \boldsymbol{f} \cdot \operatorname{curl} \psi_{h} \mathrm{~d} \boldsymbol{x}=-b\left(\mu, \psi_{h}\right)=-b\left(P_{h} \mu, \psi_{h}\right) .
$$

Using once more (2.12) leads to

$$
\int_{\Omega} \boldsymbol{f} \cdot \operatorname{curl} \psi_{h} \mathrm{~d} \boldsymbol{x}=a_{h}\left(\omega_{h}^{*}, P_{h} \mu\right)+a\left(\omega_{h}^{0}, P_{h} \mu\right)
$$

whence

$$
\left\|\omega_{h}\right\|_{L^{2}(\Omega)}+\sqrt{\alpha_{h}}\left\|\omega_{h}^{*}\right\|_{*} \leq c\left(\left\|P_{h} \mu\right\|_{L^{2}(\Omega)}+\sqrt{\alpha_{h}}\left\|P_{h} \mu\right\|_{*}+\sqrt{h_{\min }^{-1} \alpha_{h}}\|\boldsymbol{f}\|_{L^{\frac{4}{3}(\Omega)^{2}}}\right) .
$$

So, we deduce from Lemmas 3.5 and 3.6 that

$$
\left\|\omega_{h}\right\|_{L^{2}(\Omega)}+\sqrt{\alpha_{h}}\left\|\omega_{h}^{*}\right\|_{*} \leq c\left(\left(1+E_{h}\right)\|\mu\|_{W^{1, \frac{4}{3}(\Omega)}}+\sqrt{h_{\min }^{-1} \alpha_{h}}\|\boldsymbol{f}\|_{L^{\frac{4}{3}(\Omega)^{2}}}\right) .
$$

Thanks to assumption (3.21), this yields the first estimate. To establish the second one, we write

$$
\left|\psi_{h}\right|_{W^{1,4}(\Omega)}=\sup _{\boldsymbol{g} \in L^{\frac{4}{3}}(\Omega)^{2}} \frac{\int_{\Omega} \boldsymbol{g} \cdot \operatorname{grad} \psi_{h} \mathrm{~d} \boldsymbol{x}}{\|\boldsymbol{g}\|_{L^{\frac{4}{3}}(\Omega)^{2}}} .
$$

So, with any $\boldsymbol{g}$ in $L^{\frac{4}{3}}(\Omega)^{2}$, we associate the solution $\chi$ of problem (3.8), so that the function $\mu=-\Delta \chi$ belongs to $W^{1, \frac{4}{3}}(\Omega)$ and satisfies: $-\Delta \mu=\operatorname{div} \boldsymbol{g}$. This leads to

$$
\int_{\Omega} \boldsymbol{g} \cdot \operatorname{grad} \psi_{h} \mathrm{~d} \boldsymbol{x}=b\left(\mu, \psi_{h}\right)=b\left(P_{h} \mu, \psi_{h}\right),
$$

and using (2.12) gives

$$
\int_{\Omega} \boldsymbol{g} \cdot \operatorname{grad} \psi_{h} \mathrm{~d} \boldsymbol{x} \leq\left(\left\|\omega_{h}\right\|_{L^{2}(\Omega)}+\sqrt{\alpha_{h}}\left\|\omega_{h}^{*}\right\|_{*}\right)\left(\left\|P_{h} \mu\right\|_{L^{2}(\Omega)}+\sqrt{\alpha_{h}}\left\|P_{h} \mu\right\|_{*}\right) .
$$

We conclude as previously. 


\section{Extension to the Navier-Stokes equations}

We wish to extend the previous discretization to the Navier-Stokes equations. When the nonlinear convection term $(\boldsymbol{u} \cdot \nabla) \boldsymbol{u}$ is translated in terms of vorticity and stream function, they can be expressed as

$$
\begin{cases}-\Delta \omega+\frac{1}{\nu} \operatorname{curl}(\omega \operatorname{grad} \psi)=\operatorname{curl} \boldsymbol{f} & \text { in } \Omega, \\ -\Delta \psi=\omega & \text { in } \Omega, \\ \psi=\partial_{n} \psi=0 & \text { on } \partial \Omega,\end{cases}
$$

where the viscosity $\nu$ is a positive constant. Moreover, by using the previous decomposition $\omega=\omega^{0}+\omega^{*}$, this problem can equivalently be written

$$
\begin{cases}-\Delta \omega^{0}+\frac{1}{\nu} \operatorname{curl}(\omega \operatorname{grad} \psi)=\operatorname{curl} \boldsymbol{f} & \text { in } \Omega, \\ -\Delta \omega^{*}=0 & \text { in } \Omega, \\ -\Delta \psi=\omega & \text { in } \Omega, \\ \psi=\partial_{n} \psi=0 \quad \text { and } \quad \omega^{0}=0 & \text { on } \partial \Omega .\end{cases}
$$

So, in contrast to the linear problem, the equations for $\omega^{0}$ and $\omega^{*}$ are now coupled by the function $\psi$ and the full vorticity $\omega$ in the equation for $\omega^{0}$. Choosing to put the nonlinear term in the first equation relies on the idea that the main advantage of $\omega^{*}$ is to be harmonic.

We firstly write the variational formulation of this new system and we recall its properties. Next we describe the corresponding discretization. We conclude by a priori error estimates when a nonsingular solution of the continuous problem is considered.

\subsection{The continuous problem}

In order to handle the nonlinear term, we introduce the mapping $G$ defined from $L^{2}(\Omega) \times W^{1,4}(\Omega)$ into $W^{-1, \frac{4}{3}}(\Omega)$ by

$$
\forall \varphi \in W_{0}^{1,4}(\Omega), \quad\langle G(\omega, \psi), \varphi\rangle=\frac{1}{\nu} \int_{\Omega} \omega \operatorname{grad} \psi \cdot \operatorname{curl} \varphi \mathrm{d} \boldsymbol{x}
$$

where $\langle\cdot, \cdot\rangle$ now denotes the duality pairing between $W^{-1, \frac{4}{3}}(\Omega)$ and $W_{0}^{1,4}(\Omega)$. Note that this mapping is also continuous from $L^{4}(\Omega) \times W^{1,4}(\Omega)$ into $H^{-1}(\Omega)$. So, problem (4.2) admits the following equivalent variational formulation: find a triple $\left(\omega^{0}, \omega^{*}, \psi\right)$ in $W_{0}^{1, \frac{4}{3}}(\Omega) \times X \times W_{0}^{1,4}(\Omega)$ such that, with $\omega=\omega^{0}+\omega^{*}$,

$$
\begin{aligned}
\forall \eta \in W_{0}^{1,4}(\Omega), & b\left(\omega^{0}, \eta\right)+\langle G(\omega, \psi), \eta\rangle=-\int_{\Omega} \boldsymbol{f} \cdot \operatorname{curl} \eta \mathrm{d} \boldsymbol{x}, \\
\forall \theta \in X, & a\left(\omega^{*}, \theta\right)+b(\theta, \psi)=-a\left(\omega^{0}, \theta\right) \\
\forall \varphi \in W_{0}^{1,4}(\Omega), & b\left(\omega^{*}, \varphi\right)=0 .
\end{aligned}
$$

The properties of problem (4.3) are well-known, see Section 2 of Chapter IV in [12] for instance: for any data $\boldsymbol{f}$ in $L^{2}(\Omega)^{2}$, this problem has a solution and this solution is unique when the following condition holds:

$$
\frac{\|\boldsymbol{f}\|_{L^{2}(\Omega)^{2}}}{\nu^{2}} \leq \kappa
$$

where the constant $\kappa$ only depends on the domain $\Omega$.

However, in order to avoid the too restrictive assumption (4.4), we give another formulation of problem (4.3). For this, we introduce a generalized Stokes operator $S$ which associates with any data $\boldsymbol{f}$ in $L^{\frac{4}{3}}(\Omega)^{2}$, the solution 
$\left(\omega^{0}+\omega^{*}, \psi\right)$, where $\left(\omega^{0}, \omega^{*}, \psi\right)$ is the solution in $W_{0}^{1, \frac{4}{3}}(\Omega) \times X \times W_{0}^{1,4}(\Omega)$ of

$$
\begin{aligned}
& \forall \eta \in W_{0}^{1,4}(\Omega), b\left(\omega^{0}, \eta\right)=-\int_{\Omega} \boldsymbol{f} \cdot \operatorname{curl} \eta \mathrm{d} \boldsymbol{x}, \\
& \forall \theta \in X, a\left(\omega^{*}, \theta\right)+b(\theta, \psi)=-a\left(\omega^{0}, \theta\right), \\
& \forall \varphi \in W_{0}^{1,4}(\Omega), \quad b\left(\omega^{*}, \varphi\right)=0 .
\end{aligned}
$$

Indeed, we derive from the ellipticity of $a(\cdot, \cdot)$ on $V$, the inf-sup condition on $b(\cdot, \cdot)$ and some additional regularity of the function $\psi$ that this operator is well defined. Moreover, from (2.8) and (2.9), it satisfies for any data $\boldsymbol{f}$ in $L^{2}(\Omega)^{2}$,

$$
\|S \boldsymbol{f}\|_{L^{2}(\Omega) \times W^{1,4}(\Omega)} \leq c\|\boldsymbol{f}\|_{L^{2}(\Omega)^{2}},
$$

however, a further argument [10] yields the modified estimate

$$
\|S \boldsymbol{f}\|_{L^{2}(\Omega) \times W^{1,4}(\Omega)} \leq c\|\boldsymbol{f}\|_{L^{\frac{4}{3}(\Omega)^{2}}} .
$$

Next, we observe that problem (4.3) can equivalently be written:

$$
(\omega, \psi)+S \boldsymbol{F}(\omega, \psi)=0,
$$

where the mapping $\boldsymbol{F}$ is defined from $L^{2}(\Omega) \times W^{1,4}(\Omega)$ into $L^{\frac{4}{3}}(\Omega)^{2}$ by

$$
\boldsymbol{F}(\omega, \psi)=-\frac{1}{\nu} \omega \operatorname{grad} \psi-\boldsymbol{f}
$$

And we are in a position to state the key assumption on the solution.

Hypothesis 4.1. The operator $\operatorname{Id}+S D \boldsymbol{F}(\omega, \psi)$ is an isomorphism from $L^{2}(\Omega) \times W^{1,4}(\Omega)$ into itself.

From now on, we work with a solution $\left(\omega^{0}, \omega^{*}, \psi\right)$ of problem (4.3) which satisfies Hypothesis 4.1, hence is locally unique.

\subsection{The discrete problem}

The discrete problem is nearly obvious now, it reads: find a triple $\left(\omega_{h}^{0}, \omega_{h}^{*}, \psi_{h}\right)$ in $M_{h} \times X_{h} \times M_{h}$ such that, with $\omega_{h}=\omega_{h}^{0}+\omega_{h}^{*}$,

$$
\begin{aligned}
& \forall \eta_{h} \in M_{h}, \quad b\left(\omega_{h}^{0}, \eta_{h}\right)+\left\langle G\left(\omega_{h}, \psi_{h}\right), \eta_{h}\right\rangle=-\int_{\Omega} \boldsymbol{f} . \operatorname{curl} \eta_{h} \mathrm{~d} \boldsymbol{x}, \\
& \forall \theta_{h} \in X_{h}, \quad a\left(\omega_{h}^{*}, \theta_{h}\right)+b\left(\theta_{h}, \psi_{h}\right)=-a\left(\omega_{h}^{0}, \theta_{h}\right), \\
& \forall \varphi_{h} \in M_{h}, \quad b\left(\omega_{h}^{*}, \varphi_{h}\right)=0 .
\end{aligned}
$$

We intend to analyze this problem by using the discrete implicit function theorem of Brezzi, Rappaz and Raviart [8], so we must write it in a different form. In analogy with the continuous problem, we introduce the discrete Stokes operator: for any data $\boldsymbol{f}$ in $L^{\frac{4}{3}}(\Omega)^{2}, S_{h} \boldsymbol{f}$ stands for the pair $\left(\omega_{h}^{0}+\omega_{h}^{*}, \psi_{h}\right)$, where $\left(\omega_{h}^{0}, \omega_{h}^{*}, \psi_{h}\right)$ is the solution in $M_{h} \times X_{h} \times M_{h}$ of

$$
\begin{aligned}
& \forall \eta_{h} \in M_{h}, \quad b\left(\omega_{h}^{0}, \eta_{h}\right)=-\int_{\Omega} \boldsymbol{f} \cdot \operatorname{curl} \eta_{h} \mathrm{~d} \boldsymbol{x}, \\
& \forall \theta_{h} \in X_{h}, \quad a\left(\omega_{h}^{*}, \theta_{h}\right)+b\left(\theta_{h}, \psi_{h}\right)=-a\left(\omega_{h}^{0}, \theta_{h}\right), \\
& \forall \varphi_{h} \in M_{h}, \quad b\left(\omega_{h}^{*}, \varphi_{h}\right)=0 .
\end{aligned}
$$


Then, problem (4.8) can equivalently be written

$$
\left(\omega_{h}, \psi_{h}\right)+S_{h} \boldsymbol{F}\left(\omega_{h}, \psi_{h}\right)=0 .
$$

\subsection{Existence of a solution and error estimates}

Firstly, if assumptions (3.20) and (3.21) are satisfied, we recall from Corollary 3.8 the stability property

$$
\left\|S_{h} \boldsymbol{f}\right\|_{L^{2}(\Omega) \times W^{1,4}(\Omega)} \leq c\|\boldsymbol{f}\|_{L^{\frac{4}{3}}(\Omega)^{2}} .
$$

The corresponding convergence result is stated in (3.22), also when assumptions (3.20) and (3.21) hold and for any $\boldsymbol{f}$ in $L^{2}(\Omega)^{2}$ :

$$
\lim _{h \rightarrow 0}\left\|\left(S-S_{h}\right) \boldsymbol{f}\right\|_{L^{2}(\Omega) \times W^{1,4}(\Omega)}=0 .
$$

Now, let $(\omega, \psi)$ be a solution of problem (4.3) satisfying Hypothesis 4.1. The following arguments are standard.

- By writing

$$
\mathrm{Id}+S_{h} D \boldsymbol{F}(\omega, \psi)=\operatorname{Id}+S D \boldsymbol{F}(\omega, \psi)-\left(S-S_{h}\right) D \boldsymbol{F}(\omega, \psi),
$$

and noting that any solution of problem (4.3) is slightly more regular than $H^{\frac{1}{2}}(\Omega) \times H^{\frac{5}{2}}(\Omega)$ so that $D \boldsymbol{F}(\omega, \psi)$ is compact, we derive from (4.12) that $\operatorname{Id}+S_{h} D \boldsymbol{F}(\omega, \psi)$ is an isomorphism from $L^{2}(\Omega) \times W^{1,4}(\Omega)$ into itself, with the norm of its inverse bounded independently of $h$.

- Due to the improved stability property (4.11) and the continuity of the mapping: $(\theta, \chi) \mapsto \theta \cdot \operatorname{grad} \chi$ from $L^{2}(\Omega) \times W^{1,4}(\Omega)$ into $L^{\frac{4}{3}}(\Omega)^{2}$, we check that $\operatorname{Id}+S_{h} D \boldsymbol{F}(\cdot, \cdot)$ is Lipschitz-continuous in a neighbourhood of $(\omega, \psi)$, with Lipschitz constant bounded independently of $h$.

- We also observe that

$$
(\omega, \psi)+S_{h} \boldsymbol{F}(\omega, \psi)=-\left(S-S_{h}\right) \boldsymbol{F}(\omega, \psi) .
$$

Since it follows from the regularity of the solution $(\omega, \psi)$ that $\boldsymbol{F}(\omega, \psi)$ belongs to $L^{2}(\Omega)^{2}$ for any data $\boldsymbol{f}$ in $L^{2}(\Omega)^{2}$, we know from (4.13) that this last quantity tends to zero with $h$.

So applying the Brezzi-Rappaz-Raviart theorem [8] (see also Theorem 3.3 of Chapter IV in [12]) leads to the desired result.

Theorem 4.2. Assume that conditions (3.20) and (3.21) are satisfied. Then, for any solution $\left(\omega^{0}, \omega^{*}, \psi\right)$ of problem (4.3) satisfying Hypothesis 4.1 and for $h$ small enough, there exists a unique solution $\left(\omega_{h}^{0}, \omega_{h}^{*}, \psi_{h}\right)$ of problem (4.8) in a fixed neighbourhood of $\left(\omega^{0}, \omega^{*}, \psi\right)$. Moreover, this solution satisfies

$$
\left\|\omega-\omega_{h}\right\|_{L^{2}(\Omega)}+\left|\psi-\psi_{h}\right|_{W^{1,4}(\Omega)} \leq c E_{h} \sup \left\{E_{h}, 1\right\}\|\boldsymbol{f}\|_{L^{2}(\Omega)^{2}},
$$

for the quantity $E_{h}$ defined in (3.19) and a constant $c$ only depending on $\boldsymbol{f}$, and the following convergence property holds

$$
\lim _{h \rightarrow 0}\left\|\omega-\omega_{h}\right\|_{L^{2}(\Omega)}+\left|\psi-\psi_{h}\right|_{W^{1,4}(\Omega)}=0 .
$$

The estimate is exactly of the same order as for the linear problem, and the convergence property also holds with the same assumptions, that do not seem too restrictive. 


\section{Some EXtensions}

We present two extensions of the previous results. The first one also deals with affine finite elements, but on a nonconforming decomposition of the domain: it relies on the mortar element technique of Bernardi, Maday and Patera [7]. Note that this technique was firstly applied to the stream function and vorticity formulation of the Stokes problem by Ben Younes [3]. The aim of the second extension is mainly to derive an improved error estimate of type (3.18) when working with higher order finite elements.

\subsection{The mortar finite element method}

As previously, $\Omega$ denotes a bounded domain in $\mathbb{R}^{2}$ with a polygonal boundary. We now consider a fixed decomposition of $\Omega$ into a finite number of polygonal domains $\Omega_{k}$ without overlapping:

$$
\bar{\Omega}=\bigcup_{k=1}^{K} \bar{\Omega}_{k} \quad \text { and } \quad \Omega_{k} \cap \Omega_{k^{\prime}}=\emptyset, \quad 1 \leq k<k^{\prime} \leq K .
$$

We introduce the skeleton $\mathcal{S}$ of the decomposition:

$$
\mathcal{S}=\bigcup_{k=1}^{K} \partial \Omega_{k} \backslash \partial \Omega
$$

and we assume that it is a disjoint union of a finite number of "mortars" $\gamma_{m}$ :

$$
\overline{\mathcal{S}}=\bigcup_{m=1}^{M} \bar{\gamma}_{m} \quad \text { and } \quad \gamma_{m} \cap \gamma_{m^{\prime}}=\emptyset, \quad 1 \leq m<m^{\prime} \leq M,
$$

where each $\gamma_{m}$ is an edge or part of an edge of one of the polygons $\Omega_{k}$, which we denote by $\Omega_{k(m)}$. Without restriction on the geometry, we assume that the internal angles of all the corners of the $\Omega_{k}$ that do not belong to $\partial \Omega$ are $<\pi$.

On each $\Omega_{k}$, we introduce a regular family of triangulations $\left(\mathcal{T}_{h_{k}}^{k}\right)_{h_{k}}$, in the sense introduced in Section 2.2, where $h_{k}$ denotes the maximal diameter of the triangles in $\mathcal{T}_{h_{k}}^{k}$. So the global discretization parameter is a $K$-tuple of $h_{k}, 1 \leq k \leq K$, we still denote it by $h$. Indeed, the main interest of the mortar element method is that completely independent meshes can be used on the different $\Omega_{k}$, which allows firstly for handling very complex geometries, secondly for efficient mesh adaptivity (we refer to [6] for the first application of the mortar method to adaptivity and to [1] for some error indicators in the stream function and vorticity formulation). For simplicity, we make the following non restrictive assumption.

Hypothesis 5.1. For any $k, 1 \leq k \leq K$, the corners of the $\Omega_{k^{\prime}}, 1 \leq k^{\prime} \leq K$, that belong to $\partial \Omega_{k}$, are nodes of the triangulation $\mathcal{T}_{h_{k}}^{k}$ for all $h_{k}$.

Next, on each $\Omega_{k}$, the discrete space $X_{h_{k}}^{k}$ is the same as in (2.10):

$$
X_{h_{k}}^{k}=\left\{\theta_{h}^{k} \in \mathscr{C}^{0}\left(\bar{\Omega}_{k}\right) ; \forall K \in \mathcal{T}_{h_{k}}^{k}, \theta_{h \mid K}^{k} \in \mathbb{P}_{1}(K)\right\}
$$

With each edge $\Gamma$ of any $\Omega_{k}$, we also associate the space $\tilde{W}_{h_{k}}^{k}(\Gamma)$ of continuous functions which are affine on each intersection $K \cap \bar{\Gamma}, K \in \mathcal{T}_{h_{k}}^{k}$, but constant on the two intersections $K \cap \bar{\Gamma}$ that contain the endpoints of $\Gamma$.

The mortar space $\tilde{X}_{h}$ is now defined in a standard way [7]: it is the space of all functions $\theta_{h}$ such that:

- each $\theta_{h \mid \Omega_{k}}, 1 \leq k \leq K$, belongs to $X_{h_{k}}^{k}$, 
- the mortar function $\varphi$ being defined on each $\gamma_{m}, 1 \leq m \leq M$, as the trace of $\theta_{h \mid \Omega_{k(m)}}$, the following matching condition holds on all edges $\Gamma$ of the $\Omega_{k}$ :

$$
\forall \psi \in \tilde{W}_{h_{k}}^{k}(\Gamma), \quad \int_{\Gamma}\left(v_{\mid \Omega_{k}}-\varphi\right)(\tau) \psi(\tau) \mathrm{d} \tau=0
$$

And we also introduce the subspace $\tilde{M}_{h}$ of $\tilde{X}_{h}$ made of all functions which vanish on $\partial \Omega$.

Remark. Clearly the method is in general nonconforming, since neither $\tilde{M}_{h}$ is contained in $H_{0}^{1}(\Omega)$ nor $\tilde{X}_{h}$ is contained in $X$ except in some rather special situations (when all meshes are compatible and all corners of the $\Omega_{k}$ belong to $\partial \Omega$ ). So we are led to work with the following broken norms and seminorm

$$
\begin{aligned}
\|\theta\|_{\tilde{X}} & =\left(\|\theta\|_{L^{2}(\Omega)}^{2}+\sum_{k=1}^{K}\|\Delta \theta\|_{H^{-1}\left(\Omega_{k}\right)}^{2}\right)^{\frac{1}{2}} \\
\|\theta\|_{\tilde{H}^{1}(\Omega)} & =\left(\|\theta\|_{L^{2}(\Omega)}^{2}+\sum_{k=1}^{K}|\theta|_{H^{1}\left(\Omega_{k}\right)}^{2}\right)^{\frac{1}{2}} \text { and }|\theta|_{\tilde{H}^{1}(\Omega)}=\left(\sum_{k=1}^{K}|\theta|_{H^{1}\left(\Omega_{k}\right)}^{2}\right)^{\frac{1}{2}} .
\end{aligned}
$$

The definition of the form $a(\cdot, \cdot)$ remains unchanged. Now let $\mathcal{E}_{h_{k}}^{k}, 1 \leq k \leq K$, stand for the set of all edges of the $K$ in $\mathcal{T}_{h_{k}}^{k}$ which are not contained in $\partial \Omega_{k}$. We now work with a $K$-tuple of positive real numbers $\alpha_{h_{k}}^{k}$, $1 \leq k \leq K$, and we define a new bilinear form on $\tilde{X}_{h} \times \tilde{X}_{h}$ by

$$
\tilde{a}_{h}\left(\omega_{h}, \theta_{h}\right)=a\left(\omega_{h}, \theta_{h}\right)+\sum_{k=1}^{K} \alpha_{h_{k}}^{k} A_{h_{k}}^{k}\left(\omega_{h}, \theta_{h}\right),
$$

with

$$
A_{h_{k}}^{k}\left(\omega_{h}, \theta_{h}\right)=\sum_{e \in \mathcal{E}_{h_{k}}^{k}} h_{e} \int_{e}\left[\partial_{n} \omega_{h}\right]\left[\partial_{n} \theta_{h}\right] \mathrm{d} \tau
$$

We also introduce the modified bilinear form on $\tilde{X}_{h} \times \tilde{M}_{h}$ :

$$
\tilde{b}\left(\theta_{h}, \varphi_{h}\right)=-\sum_{k=1}^{K} \int_{\Omega_{k}} \operatorname{grad} \theta_{h} \cdot \operatorname{grad} \varphi_{h} \mathrm{~d} \boldsymbol{x} .
$$

Of course, when extended to $H_{0}^{1}(\Omega) \times H_{0}^{1}(\Omega)$, this form coincides with $b(\cdot, \cdot)$. The discrete problem now writes:

- firstly, find a function $\omega_{h}^{0}$ in $\tilde{M}_{h}$ such that

$$
\forall \eta_{h} \in \tilde{M}_{h}, \quad \tilde{b}\left(\omega_{h}^{0}, \eta_{h}\right)=-\sum_{k=1}^{K} \int_{\Omega_{k}} \boldsymbol{f} . \operatorname{curl} \eta_{h} \mathrm{~d} \boldsymbol{x},
$$

- secondly, find a pair $\left(\omega_{h}^{*}, \psi_{h}\right)$ in $\tilde{X}_{h} \times \tilde{M}_{h}$ such that

$$
\begin{array}{ll}
\forall \theta_{h} \in \tilde{X}_{h}, \quad \tilde{a}_{h}\left(\omega_{h}^{*}, \theta_{h}\right)+\tilde{b}\left(\theta_{h}, \psi_{h}\right)=-a\left(\omega_{h}^{0}, \theta_{h}\right), \\
\forall \varphi_{h} \in \tilde{M}_{h}, \quad \tilde{b}\left(\omega_{h}^{*}, \varphi_{h}\right)=0,
\end{array}
$$


- thirdly, set

$$
\omega_{h}=\omega_{h}^{0}+\omega_{h}^{*}
$$

The form $\tilde{a}_{h}(\cdot, \cdot)$ is still continuous and positive definite with respect to any norm on $\tilde{X}_{h}$. The form $\tilde{b}(\cdot, \cdot)$ is also continuous and satisfies a discrete inf-sup condition, see Appendix A in [7] or Proposition 2.1. in [6]. So, for any data $\boldsymbol{f}$ in $L^{2}(\Omega)^{2}$, problem (5.6)-(5.8) has a unique solution.

To derive error estimates, we must extend estimates (3.4), (3.5) and (3.7) to this new framework. The first estimate is standard in the mortar element technique (equation (5.3) in [7]), indeed the further term represents the consistency error.

Proposition 5.2. The following estimate holds

$$
\left\|\omega^{0}-\omega_{h}^{0}\right\|_{\tilde{H}^{1}(\Omega)} \leq c\left(\inf _{\eta_{h} \in \tilde{M}_{h}}\left|\omega^{0}-\eta_{h}\right|_{\tilde{H}^{1}(\Omega)}+\sup _{\mu_{h} \in \tilde{M}_{h}} \frac{\int_{\mathcal{S}}\left(\partial_{n} \omega^{0}\right)\left[\mu_{h}\right] \mathrm{d} \tau}{\left\|\mu_{h}\right\|_{\tilde{H}^{1}(\Omega)}}\right) .
$$

Next, we need an analogue of Lemma 3.1, which now concerns the kernel

$$
\tilde{V}_{h}=\left\{\theta_{h} \in \tilde{X}_{h} ; \forall \varphi_{h} \in \tilde{M}_{h}, \tilde{b}\left(\theta_{h}, \varphi_{h}\right)=0\right\}
$$

Since the new version only involves local terms (internal to each $\Omega_{k}$ ), the proof is exactly the same.

Lemma 5.3. The following estimate holds for all functions $\theta_{h}$ in $\tilde{V}_{h}$

$$
\left\|\Delta \theta_{h}\right\|_{H^{-1}\left(\Omega_{k}\right)} \leq c\left(A_{h_{k}}^{k}\left(\theta_{h}, \theta_{h}\right)\right)^{\frac{1}{2}}, \quad 1 \leq k \leq K
$$

The next idea consists in multiplying the third line in problem (2.5) by functions in $\tilde{X}_{h}$. This yields

$$
\forall \theta_{h} \in \tilde{X}_{h}, \quad a\left(\omega^{*}, \theta_{h}\right)+\tilde{b}\left(\theta_{h}, \psi\right)=-a\left(\omega^{0}, \theta_{h}\right)-\frac{1}{2} \int_{\mathcal{S}}\left(\partial_{n} \psi\right)\left[\theta_{h}\right] \mathrm{d} \tau
$$

We now derive the analogues of estimates (3.5) and (3.7) by the same arguments as in Section 3, when replacing (2.7) by (5.12). Note that the second estimate involves the modified operator $\tilde{P}_{h}$ with values in $\tilde{X}_{h}$ defined by

$$
\forall \theta_{h} \in \tilde{X}_{h}, \quad \tilde{b}\left(\mu-\tilde{P}_{h} \mu, \theta_{h}\right)=0 \quad \text { and } \quad \int_{\Omega} \tilde{P}_{h} \mu \mathrm{d} \boldsymbol{x}=\int_{\Omega} \mu \mathrm{d} \boldsymbol{x},
$$

and the broken seminorm (which now depends on the $\alpha_{h_{k}}^{k}$ )

$$
\left\|\theta_{h}\right\|_{*}^{\sim}=\left(\sum_{k=1}^{K} \alpha_{h_{k}}^{k} A_{h_{k}}^{k}\left(\theta_{h}, \theta_{h}\right)\right)^{\frac{1}{2}}
$$

We also need other broken seminorms, for $1<p<+\infty$,

$$
|\theta|_{\tilde{W}^{1, p}(\Omega)}=\left(\sum_{k=1}^{K}|\theta|_{W^{1, p}\left(\Omega_{k}\right)}^{p}\right)^{\frac{1}{p}}
$$

we denote by $\tilde{W}^{1, p}(\Omega)$ the space of functions in $L^{p}(\Omega)$ such that this seminorm is $<+\infty$. 
Proposition 5.4. The following estimates hold

$$
\begin{aligned}
\left\|\omega^{*}-\omega_{h}^{*}\right\|_{L^{2}(\Omega)}+\left\|\omega_{h}^{*}\right\|_{*}^{\sim} \leq & c\left(\left\|\omega^{0}-\omega_{h}^{0}\right\|_{L^{2}(\Omega)}+\inf _{\theta_{h} \in V_{h}}\left\{\left\|\omega^{*}-\theta_{h}\right\|_{L^{2}(\Omega)}+\left\|\theta_{h}\right\|_{*}^{\sim}\right\}\right. \\
& \left.+\sup _{\tau_{h} \in \tilde{V}_{h}} \inf _{\varphi_{h} \in \tilde{M}_{h}} \frac{\tilde{b}\left(\tau_{h}, \psi-\varphi_{h}\right)}{\left\|\tau_{h}\right\|_{L^{2}(\Omega)}+\left\|\tau_{h}\right\|_{*}}+\sup _{\tau_{h} \in \tilde{V}_{h}} \frac{\int_{\mathcal{S}}\left(\partial_{h} \psi\right)\left[\tau_{h}\right] \mathrm{d} \tau}{\left\|\tau_{h}\right\|_{L^{2}(\Omega)}}\right),
\end{aligned}
$$

and

$$
\begin{aligned}
\left|\psi-\psi_{h}\right|_{\tilde{W}^{1,4}(\Omega)} \leq & c\left(\left|\psi-\tilde{P}_{h} \psi\right|_{\tilde{W}^{1,4}(\Omega)}+\left\|\omega-\omega_{h}\right\|_{L^{2}(\Omega)} \sup _{\mu \in \tilde{W}^{1, \frac{4}{3}(\Omega)}} \frac{\left\|\mu-\tilde{P}_{h} \mu\right\|_{L^{2}(\Omega)}}{|\mu|_{\tilde{W}^{1, \frac{4}{3}}(\Omega)}}\right. \\
& \left.+\left\|\omega_{h}^{*}\right\|_{*}^{\sim} \sup _{\mu \in \tilde{W}^{1, \frac{4}{3}(\Omega)}} \frac{\left\|\tilde{P}_{h} \mu\right\|_{*}}{|\mu|_{\tilde{W}^{1, \frac{4}{3}}(\Omega)}}+\sup _{\tau_{h} \in \tilde{V}_{h}} \frac{\int_{\mathcal{S}}\left(\partial_{h} \psi\right)\left[\tau_{h}\right] \mathrm{d} \tau}{\left\|\tau_{h}\right\|_{L^{2}(\Omega)}}+\left\|\omega-\omega_{h}\right\|_{L^{2}(\Omega)}\right) .
\end{aligned}
$$

Proof. The proof of estimate (5.14) is exactly the same as for (3.5), up to the consistency error term, so we skip it over. To derive estimate (5.15), we start from

$$
\left|\psi-\psi_{h}\right|_{\tilde{W}^{1,4}(\Omega)} \leq \sup _{\boldsymbol{g}_{k} \in L^{\frac{4}{3}}\left(\Omega_{k}\right)^{2}} \sum_{k=1}^{K} \frac{1}{\left\|\boldsymbol{g}_{k}\right\|_{L^{\frac{4}{3}}\left(\Omega_{k}\right)^{2}}} \int_{\Omega_{k}} \boldsymbol{g}_{k} \cdot \operatorname{grad}\left(\psi-\psi_{h}\right) \mathrm{d} \boldsymbol{x} .
$$

So, with any function $\boldsymbol{g}_{k}$ in $L^{\frac{4}{3}}(\Omega)^{2}$ with norm 1 , we associate the solution $\mu_{k}$ in $W^{1, \frac{4}{3}}\left(\Omega_{k}\right)$, with a null integral on $\Omega_{k}$, of the problem

$$
\forall \varphi \in W^{1,4}\left(\Omega_{k}\right), \quad \int_{\Omega_{k}} \operatorname{grad} \mu_{k} \cdot \operatorname{grad} \varphi \mathrm{d} \boldsymbol{x}=\int_{\Omega_{k}} \boldsymbol{g}_{k} \cdot \operatorname{grad} \varphi \mathrm{d} \boldsymbol{x}
$$

The existence of such a solution follows from a duality argument: the operator $\Delta$ is an isomorphism [10] from $W^{1,4}\left(\Omega_{k}\right) / \mathbb{R}$ onto the orthogonal to $\mathbb{R}$ in $\left(W^{1, \frac{4}{3}}\left(\Omega_{k}\right)\right)^{\prime}$, hence from $W^{1, \frac{4}{3}}\left(\Omega_{k}\right) / \mathbb{R}$ onto the orthogonal to $\mathbb{R}$ in $\left(W^{1,4}\left(\Omega_{k}\right)\right)^{\prime}$. We define a function $\mu$ by $\mu_{\mid \Omega_{k}}=\mu_{k}, 1 \leq k \leq K$. The end of the proof relies on the same arguments as for Proposition 3.4, thanks to the new definition of the operator $\tilde{P}_{h}$, when defining $\chi_{\mid \Omega_{k}}$ as the solution of the local Dirichlet problem on $\Omega_{k}$.

It is readily checked that the analogues of Lemmas 3.5 and 3.6 still hold in our case. Estimating the consistency error terms by using the matching condition (5.4) is well known (Section V in [7]): for instance,

$$
\sup _{\mu_{h} \in \tilde{M}_{h}} \frac{\int_{\mathcal{S}}\left(\partial_{n} \omega^{0}\right)\left[\mu_{h}\right] \mathrm{d} \tau}{\left\|\mu_{h}\right\|_{\tilde{H}^{1}(\Omega)}} \leq c h^{\alpha_{i}}\left\|\omega^{0}\right\|_{H^{1+\alpha_{i}}(\Omega)} .
$$

Also, the approximation results are slightly modified, however we refer to Lemma 3.1 in [3] for the following result, valid for all functions $\mu$ in $H^{1}(\Omega)$ such that $\mu_{\mid \Omega_{k}}, 1 \leq k \leq K$, belongs to $H^{r_{k}}\left(\Omega_{k}\right), r_{k} \geq 1$,

$$
\left|\mu-\tilde{P}_{h} \mu\right|_{\tilde{H}^{1}(\Omega)} \leq c \sum_{k=1}^{K} h_{k}^{r_{k}-1}\|\mu\|_{H^{r_{k}\left(\Omega_{k}\right)}}
$$


from which the estimates in other norms can be derived. Combining all together leads to the final result. We denote by $h_{\min }$ the smallest diameter of all triangles in $\mathcal{T}_{h_{k}}^{k}, 1 \leq k \leq K$.

Theorem 5.5. The following error estimate holds between the solution $(\omega, \psi)$ of problem $(2.4)$ and the solution $\left(\omega_{h}, \psi_{h}\right)$ of problem $(5.6)-(5.8)$ :

$\left\|\omega-\omega_{h}\right\|_{L^{2}(\Omega)}+\left(\sum_{k=1}^{K} \alpha_{h_{k}}^{k}\left\|\Delta\left(\omega-\omega_{h}\right)\right\|_{H^{-1}\left(\Omega_{k}\right)}^{2}\right)^{\frac{1}{2}}+\left|\psi-\psi_{h}\right|_{\tilde{W}^{1,4}(\Omega)} \leq \sup _{1 \leq k \leq K}\left\{E_{h_{k}}^{k} \sup \left\{E_{h_{k}}^{k}, 1\right\}\right\}\|\boldsymbol{f}\|_{L^{2}(\Omega)^{2}}$,

where each $E_{h_{k}}^{k}$ denotes the quantity

$$
E_{h_{k}}^{k}=h_{\min }^{-\frac{1}{2}}\left(h_{k}+\sum_{U_{i} \cap \Omega_{k} \neq \emptyset} h_{i}^{\alpha_{i}}+\sqrt{\alpha_{h_{k}}^{k}}\right)+\frac{h_{k}}{\sqrt{\alpha_{h_{k}}^{k}}} .
$$

If assumption (3.20) holds and if the $\alpha_{h_{k}}^{k}, 1 \leq k \leq K$, are chosen such that

$$
\lim _{h \rightarrow 0} h_{\min }^{-1} \alpha_{h_{k}}^{k}=0 \quad \text { and } \quad \lim _{h \rightarrow 0} h_{k}^{2}\left(\alpha_{h_{k}}^{k}\right)^{-1}=0,
$$

the following convergence property holds:

$$
\lim _{h \rightarrow 0}\left\|\omega-\omega_{h}\right\|_{L^{2}(\Omega)}+\left(\sum_{k=1}^{K} \alpha_{h_{k}}^{k}\left\|\Delta\left(\omega-\omega_{h}\right)\right\|_{H^{-1}\left(\Omega_{k}\right)}^{2}\right)^{\frac{1}{2}}+\left|\psi-\psi_{h}\right|_{\tilde{W}^{1,4}(\Omega)}=0 .
$$

There also the estimate is exactly of the same order as in (3.18). Moreover, since local values of the $\alpha_{h_{k}}^{k}$ can be chosen, the global assumption (3.21) is replaced by a local one, so that nearly completely independent meshes can be used on the different subdomains, which allows for performing mesh adaptivity in a simple way.

\subsection{Higher order elements}

For the same domain $\Omega$ as in Section 2.2 and for an analogous regular family of triangulations $\left(\mathcal{T}_{h}\right)_{h}$, we now introduce the spaces

$$
X_{h}^{+}=\left\{\theta_{h} \in \mathscr{C}^{0}(\bar{\Omega}) ; \forall K \in \mathcal{T}_{h}, \theta_{h \mid K} \in \mathbb{P}_{\ell}(K)\right\}, \quad M_{h}^{+}=X_{h}^{+} \cap H_{0}^{1}(\Omega),
$$

where $\ell$ is an integer $\geq 2$ and $\mathbb{P}_{\ell}(K)$ denotes the space of polynomials on $K$ with degree $\leq \ell$.

For consistency reasons, we need define modified bilinear forms. So, we set

$$
a_{h}^{+}\left(\omega_{h}, \theta_{h}\right)=a\left(\omega_{h}, \theta_{h}\right)+\alpha_{h} A_{h}^{+}\left(\omega_{h}, \theta_{h}\right),
$$

with

$$
A_{h}^{+}\left(\omega_{h}, \theta_{h}\right)=\sum_{e \in \mathcal{E}_{h}} h_{e} \int_{e}\left[\partial_{n} \omega_{h}\right]\left[\partial_{n} \theta_{h}\right] \mathrm{d} \tau+\sum_{K \in \mathcal{T}_{h}} h_{K}^{2} \int_{K} \Delta \omega_{h} \Delta \theta_{h} \mathrm{~d} \boldsymbol{x}
$$

Note that the further terms in $A_{h}^{+}(\cdot, \cdot)$ vanish for $\ell=1$. Their introduction when $\ell$ is $\geq 2$ is justified later on. 
Then, for any data $\boldsymbol{f}$ in $L^{2}(\Omega)^{2}$, the discrete problem still consists in three steps:

- firstly, find a function $\omega_{h}^{0}$ in $M_{h}^{+}$such that

$$
\forall \eta_{h} \in M_{h}^{+}, \quad b\left(\omega_{h}^{0}, \eta_{h}\right)=-\int_{\Omega} \boldsymbol{f} . \operatorname{curl} \eta_{h} \mathrm{~d} \boldsymbol{x},
$$

- secondly, find a pair $\left(\omega_{h}^{*}, \psi_{h}\right)$ in $X_{h}^{+} \times M_{h}^{+}$such that

$$
\begin{array}{ll}
\forall \theta_{h} \in X_{h}^{+}, & a_{h}^{+}\left(\omega_{h}^{*}, \theta_{h}\right)+b\left(\theta_{h}, \psi_{h}\right)=-a\left(\omega_{h}^{0}, \theta_{h}\right), \\
\forall \varphi_{h} \in M_{h}^{+}, & b\left(\omega_{h}^{*}, \varphi_{h}\right)=0,
\end{array}
$$

- thirdly, set

$$
\omega_{h}=\omega_{h}^{0}+\omega_{h}^{*}
$$

For exactly the same reasons as previously, namely the positivity of $a_{h}^{+}(\cdot, \cdot)$ and the inf-sup condition on $b(\cdot, \cdot)$, this problem has a unique solution $\left(\omega_{h}, \psi_{h}\right)$ in $X_{h}^{+} \times M_{h}^{+}$.

We firstly observe that Lemma 3.1 still holds in this case, for the new discrete kernel

$$
V_{h}^{+}=\left\{\theta_{h} \in X_{h}^{+} ; \forall \varphi_{h} \in M_{h}^{+}, b\left(\theta_{h}, \varphi_{h}\right)=0\right\},
$$

and with the norm $\left\|\theta_{h}\right\|_{*}$ replaced by

$$
\left\|\theta_{h}\right\|_{*}^{+}=\left(A_{h}^{+}\left(\theta_{h}, \theta_{h}\right)\right)^{\frac{1}{2}} .
$$

Estimate (3.4) with $M_{h}$ replaced by $M_{h}^{+}$obviously holds for problem (5.11), since it is completely similar to problem (2.11). And it can also be checked that estimates (3.5) and (3.7) with further exponents + also hold (the operator $P_{h}^{+}$is still defined by (3.6) but with $X_{h}$ replaced by $X_{h}^{+}$). So it remains to analyze the terms in their right-hand sides. We still use Lemma 3.5 for the term

$$
\sup _{\mu \in W^{1, \frac{4}{3}}(\Omega)} \frac{\left\|\mu-P_{h}^{+} \mu\right\|_{L^{2}(\Omega)}}{|\mu|_{W^{1, \frac{4}{3}}(\Omega)}}
$$

and an analogue of Lemma 3.6 for the term

$$
\sup _{\mu \in W^{1, \frac{4}{3}}(\Omega)} \frac{\left\|P_{h}^{+} \mu\right\|_{L^{2}(\Omega)}}{|\mu|_{W^{1, \frac{4}{3}}(\Omega)}}
$$

(the proof of this analogous result is more technical, we skip it for the sake of brevity). However the terms concerning the approximation error are now smaller: for instance, if $\psi$ belongs to $H^{t}(\Omega), 2 \leq t \leq \ell+1$,

$$
\left|\psi-P_{h}^{+} \psi\right|_{W^{1,4}(\Omega)} \leq c h_{\min }^{-\frac{1}{2}} h^{t-1}\|\psi\|_{H^{t}(\Omega)},
$$

and, if $\omega^{*}$ belongs to $H^{s}(\Omega), \frac{1}{2} \leq s \leq \ell+1$,

$$
\inf _{\theta_{h} \in V_{h}^{+}}\left\{\left\|\omega^{*}-\theta_{h}\right\|_{L^{2}(\Omega)}+\sqrt{\alpha_{h}}\left\|\theta_{h}\right\|_{*}^{+}\right\} \leq c h_{\min }^{-\frac{1}{2}}\left(h+\sum_{i=1}^{N} h_{i}^{\alpha_{i}}+\sqrt{\alpha_{h}}\right) h^{s-\frac{1}{2}}\left\|\omega^{*}\right\|_{H^{s}(\Omega)} .
$$

Combining all this leads to the following theorem. 
Theorem 5.6. Let us assume that the solution $\left(\omega^{0}, \omega^{*}, \psi\right)$ of problem $(2.5)$ belongs to $H^{r}(\Omega) \times H^{s}(\Omega) \times H^{t}(\Omega)$ for some real numbers $r$, $s$ and $t$ such that

$$
1 \leq r \leq \ell+1, \quad \frac{1}{2} \leq s \leq \ell+1, \quad 2 \leq t \leq \ell+1
$$

The following error estimate holds between the solution $(\omega, \psi)$ of problem $(2.4)$ and the solution $\left(\omega_{h}, \psi_{h}\right)$ of problem (5.22)-(5.24):

$$
\begin{aligned}
\left\|\omega-\omega_{h}\right\|_{L^{2}(\Omega)}+\sqrt{\alpha_{h}} \| & \Delta\left(\omega-\omega_{h}\right) \|_{H^{-1}(\Omega)}+\left|\psi-\psi_{h}\right|_{W^{1,4}(\Omega)} \\
\leq & c\left(h^{r-1}+h^{s-\frac{1}{2}}+h^{t-2}\right) E_{h} \sup \left\{E_{h}, 1\right\}\left(\left\|\omega^{0}\right\|_{H^{r}(\Omega)}+\left\|\omega^{*}\right\|_{H^{s}(\Omega)}+\|\psi\|_{H^{t}(\Omega)}\right),
\end{aligned}
$$

for the quantity $E_{h}$ defined in (3.19).

Remark. Assume for a while that the solution $\left(\omega^{0}, \omega^{*}, \psi\right)$ is very smooth, i.e. that it belongs to $H^{\ell}(\Omega) \times$ $H^{\ell}(\Omega) \times H^{\ell+1}(\Omega)$. In the case $\alpha_{h}=0$ of the standard discretization, the best known convergence order in the previous norm is $h^{\ell-1}$. So, when assumptions (3.20) and (3.21) hold, the modified discretization that we propose is slightly better since the error behaves like $h^{\ell-1} E_{h}$ and $E_{h}$ tends to zero.

Remark. Let $\gamma$ denote the largest angle of $\Omega$. For smooth enough data $\boldsymbol{f}$, the solution $\omega^{0}$ of problem $(2.6)$ belongs to $H^{r_{0}+1}(\Omega)$ for all $r_{0}<\pi / \gamma$ (Section 4.4 .3 in [14]), while the solution $\left(\omega^{*}, \psi\right)$ of problem (2.7) belongs to $H^{\inf \left\{r_{0}+1, s_{0}\right\}}(\Omega) \times H^{s_{0}+2}(\Omega)$ for all $s_{0}<\eta(\gamma)$ (Section 7.3.3 in [14]), where $\eta$ is a decreasing function on ] $0,2 \pi[$. So, we have the estimate

$\left\|\omega-\omega_{h}\right\|_{L^{2}(\Omega)}+\sqrt{\alpha_{h}}\left\|\Delta\left(\omega-\omega_{h}\right)\right\|_{H^{-1}(\Omega)}+\left|\psi-\psi_{h}\right|_{W^{1,4}(\Omega)} \leq c h^{\inf \left\{r_{0}, s_{0}-\frac{1}{2}\right\}} E_{h} \sup \left\{E_{h}, 1\right\}\|\boldsymbol{f}\|_{L^{2}(\Omega)^{2}}$.

When $\Omega$ is not convex, $\eta(\gamma)$ is $<\pi / \gamma$, so that the error behaves like $h^{s_{0}-\frac{1}{2}} E_{h}$. So, working with polynomials of maximal degree $\ell=2$ leads to a better estimate than for $\ell=1$ but is more expensive, while working with polynomials of maximal degree $\ell \geq 3$ seems a priori useless.

Of course, combining the two extensions that we present in this section is possible, leading to higher order mortar element discretizations. And also both extensions can be applied to the full Navier-Stokes equations, with the same error estimates as previously.

The authors are very grateful towards Vivette Girault for her careful reading of the paper and helpful comments.

\section{REFERENCES}

[1] M. Amara, M. Ben Younes and C. Bernardi, Error indicators for the Navier-Stokes equations in stream function and vorticity formulation. Numer. Math. 80 (1998) 181-206.

[2] M. Amara and F. El Dabaghi, An optimal $\mathcal{C}^{0}$ finite element algorithm for the 2D biharmonic problem: theoretical analysis and numerical results. Numer. Math. (submitted).

[3] M. Ben Younes, Méthodes d'éléments finis avec joints pour le problème de Stokes en formulation fonction courant - tourbillon. Ph.D. thesis, Université Pierre et Marie Curie, France (1997).

[4] C. Bernardi and V. Girault, A local regularization operator for triangular and quadrilateral finite elements. SIAM J. Numer. Anal. 35 (1998) 1893-1916.

[5] C. Bernardi, V. Girault and Y. Maday, Mixed spectral element approximation of the Navier-Stokes equations in the streamfunction and vorticity formulation. IMA J. Numer. Anal. 12 (1992) 565-608.

[6] C. Bernardi and Y. Maday, Mesh adaptivity in finite elements by the mortar method. Internal Report 94029, Laboratoire d'Analyse Numérique, Université Pierre et Marie Curie, France (1994).

[7] C. Bernardi, Y. Maday and A.T. Patera, A new nonconforming approach to domain decomposition: the mortar element method, in Collège de France Seminar XI, H. Brezis and J.-L. Lions Eds., Pitman (1994) 13-51. 
[8] F. Brezzi, J. Rappaz and P.-A. Raviart, Finite dimensional approximation of nonlinear problems, Part I: Branches of nonsingular solutions. Numer. Math. 36 (1980) 1-25.

[9] P.G. Ciarlet, Basic Error Estimates for Elliptic Problems, in Handbook of Numerical Analysis, Vol. II, P.G. Ciarlet and J.-L. Lions Eds., North-Holland (1991).

[10] M. Dauge, Neumann and mixed problems on curvilinear polyhedra. Integral Equations Operator Theory 15 (1992) $227-261$.

[11] V. Girault and P.-A. Raviart, An analysis of a mixed finite element method for the Navier-Stokes equations. Numer. Math. 33 (1979) 235-271.

[12] V. Girault and P.-A. Raviart, Finite Element Methods for the Navier-Stokes Equations, Theory and Algorithms. SpringerVerlag (1986).

[13] R. Glowinski and O. Pironneau, Numerical methods for the first biharmonic equation and for the two-dimensional Stokes problem. SIAM Rev. 21 (1979) 167-212.

[14] P. Grisvard, Elliptic Problems in Nonsmooth Domains. Pitman (1985).

[15] R. Scholz, A mixed method for fourth order problems using linear finite elements. RAIRO Anal. Numér. 12 (1978) 85-90. 